\title{
A CLASS OF COMPUTATIONALLY FAST FIRST ORDER FINITE VOLUME SOLVERS: PVM METHODS.*
}

\author{
M. J. CASTRO DíAZ † AND E. D. FERNÁNDEZ-NIETO ‡
}

\begin{abstract}
In this work, we present a class of fast first order finite volume solvers, named as PVM (Polynomial Viscosity Matrix), for balance laws or, more generally, for nonconservative hyperbolic systems. They are defined in terms of viscosity matrices computed by a suitable polynomial evaluation of a Roe matrix. These methods have the advantage that they only need some information about the eigenvalues of the system to be defined, and no spectral decomposition of Roe Matrix is needed. As consequence, they are faster than Roe method. These methods can be seen as a generalization of the schemes introduced by Degond et al. in [12] for balance laws and nonconservative systems. The first-order path conservative methods to be designed here are intended to be used as the basis for higher order methods for multi-dimensional problems. In this work, some well known solvers as Rusanov, Lax-Friedrichs, FORCE (see [30], [8]), GFORCE (see [31], [8]) or HLL (see [18]) are redefined under this form, and then some new solvers are proposed. Finally, some numerical tests are presented and the performance of the numerical schemes are compared among them and with Roe scheme.
\end{abstract}

Key words. Finite Volume Method, path-conservative, FORCE, GFORCE, HLL, PVM methods.

AMS subject classifications. 65N06, 76B15, 76M20, 76N99.

1. Introduction. The goal of this article is to design robust, simple and fast first order explicit numerical schemes for solving Cauchy problems for hyperbolic systems of conservation laws with source terms and/or nonconservative products:

$$
w_{t}+F(w)_{x}+B(w) \cdot w_{x}=G(w) H_{x},
$$

where $w(x, t)$ takes values on an open convex set $\mathcal{O} \subset \mathbb{R}^{N}, F$ is a regular function from $\mathcal{O}$ to $\mathbb{R}^{N}, B$ is a regular matrix function from $\mathcal{O}$ to $\mathcal{M}_{N \times N}(\mathbb{R}), G$ is a function from $\mathcal{O}$ to $\mathbb{R}^{N}$, and $H$ is a function from $\mathbb{R}$ to $\mathbb{R}$. A number of models of this type have been introduced in fluid dynamics to serve as simplified models of multiphase or multilayer flows.

The theory introduced in [10] is used here to define the weak solutions of the system (1.1). This theory allows one to give a sense to the nonconservative terms of the system as Borel measures provided that a Lispchitz-continuous family of paths is prescribed in the space of states.

We consider here the discretization of system (1.1) by means of numerical schemes which are path-conservative in the sense introduced in [24]. The concept of pathconservative method, which is also based on a prescribed family of paths, provides a generalization of conservative schemes introduced by Lax for systems of conservation laws. The first-order path conservative methods to be designed here are intended to be used as the basis for higher order methods for multi-dimensional problems.

In [6] and [25] it has been proved that, in general, the numerical solutions provided by a path-conservative numerical scheme converge to functions which solve a

*This research has been partially supported by the Spanish Government Research projects MTM2009-11923 and P06-RNM-01594. The numerical computations have been performed at the Laboratory of Numerical Methods of the University of Málaga.

†Dpto. Análisis Matemático, Universidad de Málaga, Campus Teatinos s/n, 29071 Málaga, Spain (castro@anamat.cie.uma.es)

${ }_{\ddagger}^{\ddagger}$ Departamento de Matemática Aplicada I, Universidad de Sevilla. E.T.S. Arquitectura. Avda, Reina Mercedes, s/n. 41012 Sevilla, Spain (edofer@us.es) 
perturbed system in which an error source-term appears on the right-hand side. The appearance of this source term, which is a measure supported on the discontinuities, has been first observed in [19] when a scalar conservation law is discretized by means of a nonconservative numerical method. Nevertheless, in certain special situations the convergence error vanishes for finite difference methods: this is the case for systems of balance laws (see [23]). Moreover for more general problems, even when the convergence error is present, it may be only noticeable for very fine meshes, for discontinuities of large amplitude, and/or for large-time simulations: see [6], [25] for details.

The family of generalized Roe schemes introduced in [32] constitutes a particular case of path-conservative numerical methods. Although the schemes of this family are robust and have good well-balanced properties (see, for instance [2],[7],[26],[24]) they also present, as their conservative counterpart, some drawbacks as their implementation requires the explicit knowledge of the eigenstructure of the intermediate matrices (see [29]). Some times their analytic expression is not available, making Roe schemes computationally expensive. Also, they do not satisfy in general an entropy inequality, as a consequence, an entropy-fix technique has to be added to capture the entropy solution in the presence of smooth transitions (see [17]). It is also well known that the use of incomplete Riemann solvers as Rusanov, Lax-Friedrichs, HLL, etc. allows one to reduce the cpu time required by a Roe solver which resolves all the characteristic fields (see, for instance, [15]). Although when combined with piecewise constant approximation Roe solvers give in general a better resolution of the discontinuities than incomplete Riemann solvers, when combined with high order reconstructions the resolution may be indistinguishable. Therefore high order methods based on incomplete Riemann solvers may be more efficient than high order Roe methods.

The goal of this article is to design incomplete simple Riemann solvers named as PVM (Polynomial Viscosity Matrix), for nonconservative hyperbolic systems, defined in terms of viscosity matrices computed by a suitable polynomial evaluation of a Roe linearization, that overcome theses difficulties. PVM schemes can be seen as the natural extension of the one proposed in [12] for balance laws, and, more generally, for nonconservative systems.

The structure of this paper is the following: In Section 2 we recall some basic concepts. Section 3 is the core of the article. PVM schemes are introduced for balance laws and for nonconservative hyperbolic systems. First, some well known solvers as Rusanov, Lax-Friedrichs, FORCE (see [30], [8]), GFORCE (see [31], [8]) or HLL (see [18]) are redefined under the form of a PVM scheme, and then some new solvers are proposed. Next, a simple analysis of the numerical diffusion associated to the considered schemes are provided. Finally, the extension to high order is performed, following the main ideas introduced in [3].

In Section 4 the numerical schemes introduced here are compared between them and with Roe scheme when they are applied to the one-layer shallow water system and the two-fluid flow model of Pitman and Le [28] under the formulation given by Pelanti et al. in [27], where the friction terms are neglected. Finally, some conclusions are derived.

2. Preliminaries. Let us consider system (1.1). By adding to (1.1) the equation $H_{t}=0$, the system (1.1) can be rewritten under the form

$$
W_{t}+\mathcal{A}(W) \cdot W_{x}=0,
$$


where $W$ is the augmented vector

$$
W=\left[\begin{array}{c}
w \\
H
\end{array}\right] \in \Omega=\mathcal{O} \times \mathbb{R} \subset \mathbb{R}^{N+1}
$$

and $\mathcal{A}(W)$ is the matrix whose block structure is given by:

$$
\mathcal{A}(W)=\left[\begin{array}{c|c}
A(w) & -G(w) \\
\hline 0 & 0
\end{array}\right],
$$

where $A(w)=J(w)+B(w)$ and $J(w)=\frac{\partial F}{\partial w}(w)$.

Solutions of (2.1) may develop discontinuities and, due to the non-divergence form of the equations, the notion of weak solution in the sense of distributions cannot be used. Under some hypotheses of regularity for $\mathcal{A}$, the theory introduced by Dal Maso, LeFloch, and Murat (DLM in the former) [10] allows one to define the nonconservative product $\mathcal{A}(W) W_{x}$ as a bounded measure for functions $W$ with bounded variation, which may have step-like singularities, provided a family of Lipschitz continuous paths, $\Phi\left(s ; W_{L}, W_{R}\right), s \in[0,1]$, is prescribed, which must satisfy certain regularity and compatibility conditions, in particular

$$
\Phi\left(0 ; W_{L}, W_{R}\right)=W_{L}, \quad \Phi\left(1 ; W_{L}, W_{R}\right)=w_{R}, \quad \Phi(s ; W, W)=W .
$$

The interested reader is addressed to [10] for a rigourous and complete presentation of this theory. Here, the family of paths will be just understood as a tool to give a sense to integrals of the form:

$$
\int_{a}^{b} \mathcal{A}(W(x)) W_{x}(x) d x
$$

for functions $W$ with jump discontinuities. More precisely, given a bounded variation function $W:[a, b] \rightarrow \mathbb{R}^{N+1}$, we define:

$$
\begin{array}{rl}
f_{a}^{b} \mathcal{A}(W(x)) W_{x}(x) d x=\int_{a}^{b} & \mathcal{A}(W(x)) W_{x}(x) d x \\
& +\sum_{m} \int_{0}^{1} \mathcal{A}\left(\Phi\left(s ; W_{m}^{-}, W_{m}^{+}\right)\right) \frac{\partial \Phi}{\partial s}\left(s ; W_{m}^{-}, W_{m}^{+}\right) d s
\end{array}
$$

In this definition, $W_{m}^{-}$and $W_{m}^{+}$represent, respectively, the limits of $W$ to the left and right of its $m$-th discontinuity. Observe that, in (2.3), the family of paths has been used to determine the Dirac measures placed at the discontinuities of $W$, and the first term on the RHS is the integral computed in regions of smoothness of $W$, in the usual sense.

Notice that the meaning of the nonconservative products and thus the concept of weak solution has to be assigned together with the system of equations, and its initial/boundary conditions. For example, a family of straight segments can be considered:

$$
\Phi\left(s ; W_{L}, W_{R}\right)=W_{L}+s\left(W_{R}-W_{L}\right) .
$$

A concept of entropy is also necessary to select the meaningful physical solutions as it occurs in systems of conservation laws. 
We consider here path-conservative numerical schemes in the sense defined in [24], that is, numerical schemes of the general form:

$$
W_{i}^{n+1}=W_{i}^{n}-\frac{\Delta t}{\Delta x}\left(\mathcal{D}_{i-1 / 2}^{+}+\mathcal{D}_{i+1 / 2}^{-}\right),
$$

where $\Delta x$ is, for simplicity, assumed to be constant; $W_{i}^{n}$ is the approximation provided by the numerical scheme of the cell average of the exact solution at the $i$-th cell, $I_{i}=\left[x_{i-1 / 2}, x_{i+1 / 2}\right]$ at the $n$-th time level $t^{n}=n \Delta t$, and

$$
\mathcal{D}_{i+1 / 2}^{ \pm}=\mathcal{D}^{ \pm}\left(W_{i}^{n}, W_{i+1}^{n}\right)
$$

where $\mathcal{D}^{-}$and $\mathcal{D}^{+}$are two Lipschitz continuous functions from $\Omega \times \Omega$ to $\Omega$ satisfying:

$$
\mathcal{D}^{ \pm}(W, W)=0, \quad \forall W \in \Omega,
$$

and for every $W_{L}, W_{R} \in \Omega$,

$$
\mathcal{D}^{-}\left(W_{L}, W_{R}\right)+\mathcal{D}^{+}\left(W_{L}, W_{R}\right)=\int_{0}^{1} \mathcal{A}\left(\Phi\left(s ; W_{L}, W_{R}\right)\right) \frac{\partial \Phi}{\partial s}\left(s ; W_{L}, W_{R}\right) d s .
$$

These conditions provide a generalization of the concept of conservative scheme introduced by Lax for systems of conservation laws.

Let us remark that the two main difficulties linked to the discretization of nonconservative systems using the DLM theory, that is, the choice of the family of paths and the convergence of the numerical solutions, are not studied in this paper. We refer to $[25]$ for a review.

The numerical schemes considered in this work are based on a decomposition of a generalized Roe matrix for (2.1) defined by Toumi in [32]: given a family of paths $\Phi$, a function $\mathcal{A}_{\Phi}: \Omega \times \Omega \mapsto \mathcal{M}_{(N+1) \times(N+1)}(\mathbb{R})$ is called a Roe linearization if it verifies the following properties:

- for any $W_{L}, W_{R} \in \Omega, \mathcal{A}_{\Phi}\left(W_{L}, W_{R}\right)$ has $N+1$ distinct real eigenvalues,

- for every $W \in \Omega, \mathcal{A}_{\Phi}(W, W)=\mathcal{A}(W)$;

- for any $W_{L}, W_{R} \in \Omega$,

$$
\mathcal{A}_{\Phi}\left(W_{L}, W_{R}\right) \cdot\left(W_{R}-W_{L}\right)=\int_{0}^{1} \mathcal{A}\left(\Phi\left(s ; W_{L}, W_{R}\right)\right) \frac{\partial \Phi}{\partial s}\left(s ; W_{L}, W_{R}\right) d s .
$$

Following [26], we consider Roe linearizations for system (1.1) given by:

$$
\mathcal{A}_{\Phi}\left(W_{L}, W_{R}\right)=\left[\begin{array}{c|c}
A_{\Phi}\left(W_{L}, W_{R}\right) & -G_{\Phi}\left(W_{L}, W_{R}\right) \\
\hline 0 & 0
\end{array}\right]
$$

where

$$
A_{\Phi}\left(W_{L}, W_{R}\right)=\mathcal{L}\left(w_{L}, w_{R}\right)+B_{\Phi}\left(W_{L}, W_{R}\right) .
$$

Here, $\mathcal{L}\left(w_{L}, w_{R}\right)$ is a Roe linearization of the Jacobian of the flux $F$ in the usual sense:

$$
\mathcal{L}\left(w_{L}, w_{R}\right) \cdot\left(w_{R}-w_{L}\right)=F\left(w_{R}\right)-F\left(w_{L}\right) ;
$$

$B_{\Phi}\left(W_{L}, W_{R}\right)$ is a matrix satisfying:

$$
B_{\Phi}\left(W_{L}, W_{R}\right) \cdot\left(w_{R}-w_{L}\right)=\int_{0}^{1} B\left(\Phi\left(s ; W_{L}, W_{R}\right)\right) \frac{\partial \Phi_{w}}{\partial s}\left(s ; W_{L}, W_{R}\right) d s
$$


and $G_{\Phi}\left(W_{L}, W_{R}\right)$ is a vector satisfying:

$$
G_{\Phi}\left(W_{L}, W_{R}\right)\left(H_{R}-H_{L}\right)=\int_{0}^{1} G\left(\Phi\left(s ; W_{L}, W_{R}\right)\right) \frac{\partial \Phi_{H}}{\partial s}\left(s ; W_{L}, W_{R}\right) d s .
$$

It can be easily shown that, if (2.9)-(2.11) are fulfilled, then the matrix defined by (2.7)-(2.8) is thus a Roe linearization provided it has $N+1$ different real eigenvalues.

Once the Roe linearization has been chosen, a numerical scheme can be defined by (2.4) with

$$
\mathcal{D}_{i+1 / 2}^{ \pm}=\widehat{\mathcal{A}}_{\Phi}^{ \pm}\left(W_{i}^{n}, W_{i+1}^{n}\right) \cdot\left(W_{i+1}^{n}-W_{i}^{n}\right)
$$

where

$$
\mathcal{A}_{\Phi}\left(W_{L}, W_{R}\right)=\widehat{\mathcal{A}}_{\Phi}^{+}\left(W_{L}, W_{R}\right)+\widehat{\mathcal{A}}_{\Phi}^{-}\left(W_{L}, W_{R}\right)
$$

is any decomposition of the Roe linearization of the form:

$$
\widehat{\mathcal{A}}_{\Phi}^{ \pm}\left(W_{L}, W_{R}\right)=\frac{1}{2}\left(\mathcal{A}_{\Phi}\left(W_{L}, W_{R}\right) \pm Q_{\Phi}\left(W_{L}, W_{R}\right)\right),
$$

where $\mathcal{Q}_{\Phi}\left(W_{L}, W_{R}\right)$ is defined as follows

$$
\mathcal{Q}_{\Phi}\left(W_{L}, W_{R}\right)=\left[\begin{array}{c|c}
Q_{\Phi}\left(W_{L}, W_{R}\right) & -Q_{\Phi}\left(W_{L}, W_{R}\right) A_{\Phi}^{-1}\left(W_{L}, W_{R}\right) G_{\Phi}\left(W_{L}, W_{R}\right) \\
\hline 0 & 0
\end{array}\right]
$$

where $Q_{\Phi}\left(W_{L}, W_{R}\right)$ is a numerical viscosity matrix defined using $A_{\Phi}\left(W_{L}, W_{R}\right)$.

Therefore, different numerical schemes can be obtained choosing different viscosity matrices. For example, Roe scheme corresponds to the choice

$$
Q_{\Phi}\left(W_{L}, W_{R}\right)=\left|A_{\Phi}\left(W_{L}, W_{R}\right)\right|
$$

where

$$
\left|A_{\Phi}\left(W_{L}, W_{R}\right)\right|=\mathcal{K}_{\Phi}\left(W_{L}, W_{R}\right)\left|\Lambda_{\Phi}\left(W_{L}, W_{R}\right)\right| \mathcal{K}_{\Phi}^{-1}\left(W_{L}, W_{R}\right),
$$

where $\left|\Lambda_{\Phi}\left(W_{L}, W_{R}\right)\right|$ the diagonal matrix whose coefficients are the absolute value of the eigenvalues of $A_{\Phi}\left(W_{L}, W_{R}\right)$ and $\mathcal{K}_{\Phi}\left(W_{L}, W_{R}\right)$ is the matrix whose $i$-th column is an eigenvector associated to the $i$-th eigenvalue.

Lax-Friedrichs scheme corresponds to the choice

$$
Q_{\Phi}\left(W_{L}, W_{R}\right)=\frac{\Delta x}{\Delta t} I d
$$

where $I d$ the identity matrix.

FORCE and GFORCE schemes (see [30], [31] and [8]), correspond to the choice

$$
Q_{\Phi}\left(W_{L}, W_{R}\right)=(1-\omega) \frac{\Delta x}{\Delta t} I d+\omega \frac{\Delta t}{\Delta x} A_{\Phi}^{2}\left(W_{L}, W_{R}\right),
$$

with $\omega=0.5$ and $\omega=\frac{1}{1+\gamma}$, respectively, where $\gamma$ the CFL parameter (3.4).

Finally, the numerical scheme in the unknowns $w$ can be written as follows:

$$
w_{i}^{n+1}=w_{i}^{n}-\frac{\Delta t}{\Delta x}\left(D_{i-1 / 2}^{+}+D_{i+1 / 2}^{-}\right),
$$


where $D_{i+1 / 2}^{ \pm}=D\left(w_{i}, w_{i+1}, H_{i}, H_{i+1}\right)^{ \pm}$

$$
\begin{aligned}
D\left(w_{i}, w_{i+1}, H_{i}, H_{i+1}\right)^{ \pm}= & \frac{1}{2}\left(F\left(w_{i+1}\right)-F\left(w_{i}\right)+B_{i+1 / 2}\left(w_{i+1}-w_{i}\right)\right. \\
& -G_{i+1 / 2}\left(H_{i+1}-H_{i}\right) \\
& \left. \pm Q_{i+1 / 2}\left(w_{i+1}-w_{i}-A_{i+1 / 2}^{-1} G_{i+1 / 2}\left(H_{i+1}-H_{i}\right)\right)\right),
\end{aligned}
$$

where $B_{i+1 / 2}=B_{\Phi}\left(W_{i}, W_{i+1}\right), G_{i+1 / 2}=G_{\Phi}\left(W_{i}, W_{i+1}\right)$ and $A_{i+1 / 2}=A_{\Phi}\left(W_{i}, W_{i+1}\right)$ and $Q_{i+1 / 2}=Q_{\Phi}\left(W_{i}, W_{i+1}\right)$.

Notice that in the definition of (2.21) the term

$$
C_{i+1 / 2}=Q_{i+1 / 2} A_{i+1 / 2}^{-1} G_{i+1 / 2}\left(H_{i+1}-H_{i}\right)
$$

can be interpreted as the upwinding part of the source term discretization, and it makes no sense if one of the eigenvalues of $A_{i+1 / 2}$ vanishes. In this case, two eigenvalues of $\mathcal{A}_{\Phi}\left(W_{i}^{n}, W_{i+1}^{n}\right)$ vanish and the problem is said to be resonant. Resonant problems exhibit an additional difficulty, as weak solutions may not be uniquely determined by their initial data. The analysis of this difficulty is beyond the scope of this article. Here, we follow the strategy described in [8] to get rid of this difficulty and to obtain well-balanced numerical schemes for a given set of stationary solutions.

In this paper we focus into the definition of $Q_{\Phi}\left(W_{L}, W_{R}\right)$. In particular, we propose a family of methods, denoted by PVM methods, in which, $Q_{\Phi}$ is defined by polynomial evaluation of $A_{\Phi}$.

3. PVM methods. We propose a class of finite volume methods defined by (2.20)-(2.21), where the numerical viscosity matrix is given by

$$
Q_{i+1 / 2}=P_{l}^{i+1 / 2}\left(A_{i+1 / 2}\right),
$$

where $P_{l}^{i+1 / 2}(x)$ a polynomial of degree $l$,

$$
P_{l}^{i+1 / 2}(x)=\sum_{j=0}^{l} \alpha_{j}^{i+1 / 2} x^{j}
$$

and $A_{i+1 / 2}=A_{\Phi}\left(W_{i}, W_{i+1}\right)$ a Roe matrix.

Taking into account (3.1) and using (2.8)-(2.9), $D_{i+1 / 2}^{ \pm}$can be rewritten as follows:

$$
\begin{aligned}
D_{i+1 / 2}^{ \pm} & =\frac{ \pm \alpha_{0}^{i+1 / 2}}{2}\left(w_{i+1}-w_{i}-A_{i+1 / 2}^{-1} G_{i+1 / 2}\left(H_{i+1}-H_{i}\right)\right) \\
& +\sum_{j=1}^{l} \frac{\delta_{j, 1} \pm \alpha_{j}^{i+1 / 2}}{2} A_{i+1 / 2}^{(j-1)}\left(F\left(w_{i+1}\right)-F\left(w_{i}\right)+B_{i+1 / 2}\left(w_{i+1}-w_{i}\right)\right) \\
& -\sum_{j=1}^{l} \frac{\delta_{j, 1} \pm \alpha_{j}^{i+1 / 2}}{2} A_{i+1 / 2}^{(j-1)} G_{i+1 / 2}\left(H_{i+1}-H_{i}\right),
\end{aligned}
$$

where

$$
\delta_{j, 1}= \begin{cases}1 & \text { if } j=1 \\ 0 & \text { otherwise. }\end{cases}
$$


The definition of the polynomial (3.2) will be related to the stability and the numerical diffusion of the scheme. Moreover, if $\lambda_{1, i+1 / 2}<\lambda_{2, i+1 / 2}<\cdots<\lambda_{N, i+1 / 2}$ are the eigenvalues of $A_{i+1 / 2}$, then the numerical scheme (2.20)-(2.21)-(3.3) is linearly $\mathrm{L}^{\infty}$-stable under the usual CFL condition

$$
\frac{\Delta t}{\Delta x} \max _{i, j}\left|\lambda_{j, i+1 / 2}\right|=\gamma \leq 1 .
$$

A sufficient condition to ensure that the numerical scheme is linearly $L^{\infty}$-stable is that (see [12])

$$
\gamma \frac{\Delta x}{\Delta t} \geq P_{l}^{i+1 / 2}(x) \geq|x| \quad \forall x \in\left[\lambda_{1, i+1 / 2}, \lambda_{N, i+1 / 2}\right], \forall i \in \mathbb{Z} .
$$

Effectively, note that for linear problems the numerical scheme (2.20), (2.21), (3.3) can be written as

$$
\begin{aligned}
w_{i}^{n+1}= & \left(I d-\frac{\Delta t}{\Delta x} \frac{P_{l}^{i-1 / 2}\left(A_{i-1 / 2}\right)+P_{l}^{i+1 / 2}\left(A_{i+1 / 2}\right)}{2}\right) w_{i}^{n}- \\
& -\frac{\Delta t}{\Delta x} \frac{P_{l}^{i-1 / 2}\left(A_{i-1 / 2}\right)-A_{i-1 / 2}}{2} w_{i-1}^{n}+\frac{\Delta t}{\Delta x} \frac{P_{l}^{i+1 / 2}\left(A_{i+1 / 2}\right)-A_{i+1 / 2}}{2} w_{i+1}^{n} .
\end{aligned}
$$

The condition $P_{l}^{i+1 / 2}(x) \geq|x|, \forall x \in\left[\lambda_{1, i+1 / 2}, \lambda_{N, i+1 / 2}\right]$ implies that

$$
\left[\mathcal{K}_{i+1 / 2}^{-1}\left(P_{l}^{i+1 / 2}\left(A_{i+1 / 2}\right)-A_{i+1 / 2}\right) \mathcal{K}_{i+1 / 2}\right]_{j j} \geq 0, \quad \forall j=1, \ldots, N,
$$

where $\mathcal{K}_{i+1 / 2}=\mathcal{K}_{\Phi}\left(w_{i}, w_{i+1}\right)$. On the other hand, the condition $P_{l}^{i+1 / 2}(x) \leq \gamma \frac{\Delta x}{\Delta t}$ implies that

$$
\left[\mathcal{K}_{i+1 / 2}^{-1}\left(I d-\frac{\Delta t}{\Delta x} P_{l}^{i+/ 2}\left(A_{i+1 / 2}\right)\right) \mathcal{K}_{i+1 / 2}\right]_{j j} \geq 0, \quad \forall j=1, \ldots, N .
$$

Then, (3.5) implies that the numerical scheme is linearly $\mathrm{L}^{\infty}$-stable under the usual CFL condition.

Let us consider the following notation: a numerical scheme (2.20)-(2.21)-(3.3) whose viscosity matrix $Q_{i+1 / 2}$ is defined in terms of the polynomial $P_{l}^{i+1 / 2}(x)$ which coefficients depend on the choice of some parameters $S_{0}, \cdots, S_{k}$ will be denoted by PVM-l $\left(S_{0}, \cdots, S_{k}\right)$. In practice, the parameters $S_{0}, \cdots, S_{k}$ will be related to the approximations of some wave speeds. Thus, for example, Lax-Friedrichs corresponds to $\operatorname{PVM}-0\left(S_{0}\right)$, where $S_{0}=\frac{\Delta x}{\Delta t}$, where $\Delta t$ is related to the maximum wave speed by the CFL condition (3.4).

Definition 3.1. A PVM method is said to be upwind if

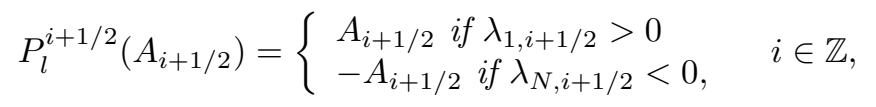

and it will be denoted as $P V M-l U$. Thus, it is straightforward that if

$$
P_{l}^{i+1 / 2}(x)=\left\{\begin{array}{l}
x \text { if } \lambda_{1, i+1 / 2}>0 \\
-x \text { if } \lambda_{N, i+1 / 2}<0,
\end{array} \quad i \in \mathbb{Z},\right.
$$

the resulting PVM is an upwind scheme.

REMARK 1. If $\alpha_{1}=0$, then the resulting PVM method is not an upwind scheme. 
REMARK 2. In what follows, in order to simplify the notation, we drop the dependency of $i+1 / 2$ on the definition of the polynomials and its coefficients. That is, we denote $P_{l}(x)$ instead of $P_{l}^{i+1 / 2}(x)$ and $\alpha_{j}$ instead of $\alpha_{j}^{i+1 / 2}$.

In what follows, some PVM schemes are derived. Some of them like Rusanov, Lax-Friedrichs, FORCE (see [30], [8]), GFORCE (see [31], [8]) or HLL (see [18]) are redefined under this form, and some new solvers are also proposed.

3.1. PVM-(N-1)U $\left(\lambda_{1}, \cdots, \lambda_{n}\right)$ or Roe method. Roe method corresponds to the choice $Q_{\Phi}\left(W_{L}, W_{R}\right)=\left|A_{\Phi}\left(W_{L}, W_{R}\right)\right|$, where $\left|A_{\Phi}\left(W_{L}, W_{R}\right)\right|$ is given by $(2.17)$. Notice that, in order to rewrite Roe method as a PVM method, $\left|A_{\Phi}\left(W_{L}, W_{R}\right)\right|$ can be redefined as

$$
\left|A_{\Phi}\left(W_{L}, W_{R}\right)\right|=\sum_{j=0}^{N-1} \alpha_{j} A_{\Phi}^{j}\left(W_{L}, W_{R}\right)
$$

where $\alpha_{j}, j=0, \cdots, N-1$ are the solution of the following linear system:

$$
\left(\begin{array}{cccc}
1 & \lambda_{1} & \ldots & \lambda_{1}^{N-1} \\
1 & \lambda_{2} & \ldots & \lambda_{2}^{N-1} \\
\vdots & \vdots & \ddots & \vdots \\
1 & \lambda_{N} & \cdots & \lambda_{N}^{N-1}
\end{array}\right)\left(\begin{array}{c}
\alpha_{0} \\
\alpha_{1} \\
\vdots \\
\alpha_{N-1}
\end{array}\right)=\left(\begin{array}{c}
\left|\lambda_{1}\right| \\
\left|\lambda_{2}\right| \\
\vdots \\
\left|\lambda_{N}\right|
\end{array}\right)
$$

where $\lambda_{1}, \cdots, \lambda_{N}$ are the eigenvalues of the matrix $A_{\Phi}\left(W_{L}, W_{R}\right)$. Notice that system (3.8) has a unique solution provided that all eigenvalues are different. Therefore, Roe method can be redefined as a PVM- $(\mathrm{N}-1) \mathrm{U}\left(\lambda_{1}, \cdots, \lambda_{N}\right)$ where $Q_{\Phi}=P_{N-1}\left(A_{\Phi}\right)$, and $P_{N-1}(x)$ is the polynomial whose coefficients are given by (3.8).

3.2. PVM-0 $\left(S_{0}\right)$ methods: Rusanov, Lax-Friedrichs and modified LaxFriedrichs schemes. The simplest choice for a PVM method corresponds to

$$
P_{0}(x)=S_{0}
$$

That is, $y=P_{0}(x)$ is an horizontal line (see Figure 3.1). Stability requirements imply that $\max _{j}\left|\lambda_{j, i+1 / 2}\right| \leq S_{0} \leq \frac{\Delta x}{\Delta t}$. Thus, several interesting choices for $S_{0}$ can be performed, taking into account that $\max _{j}\left|\lambda_{j, i+1 / 2}\right|=\gamma \frac{\Delta x}{\Delta t} \leq \frac{\Delta x}{\Delta t}$. Therefore, $S_{0}$ can be defined by

$$
S_{0} \in\left\{S_{R u s}, S_{L F}, S_{L F}^{m o d}\right\}
$$

where $S_{R u s}=\max _{j}\left|\lambda_{j, i+1 / 2}\right|, S_{L F}=\frac{\Delta x}{\Delta t}$ and $S_{L F}^{m o d}=\gamma \frac{\Delta x}{\Delta t}$. Notice that Rusanov scheme corresponds to the choice $S_{0}=S_{R u s}$, Lax-Friedrichs to $S_{0}=S_{L F}$ and modified Lax-Friedrichs to $S_{0}=S_{L F}^{\bmod }$.

3.3. PVM-1U $\left(S_{L}, S_{R}\right)$ or HLL method. The definition of the classical HLL flux (see [18]) for a conservative system can be written as follows

$$
w_{i}^{n+1}=w_{i}^{n}-\frac{\Delta t}{\Delta x}\left(\mathcal{F}_{i+1 / 2}^{H L L}-\mathcal{F}_{i-1 / 2}^{H L L}\right)
$$




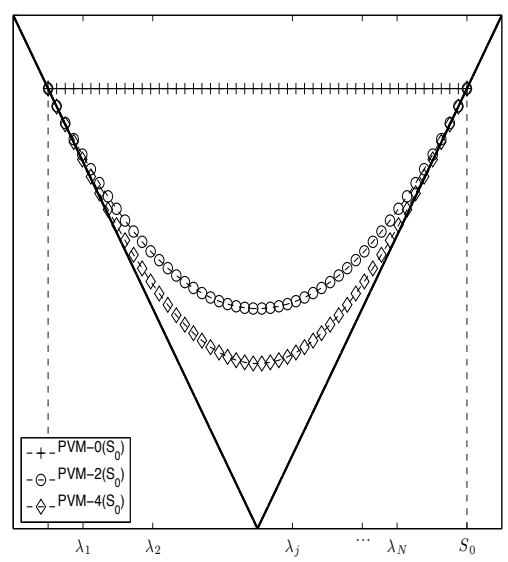

(a) one-wave PVM polynomials

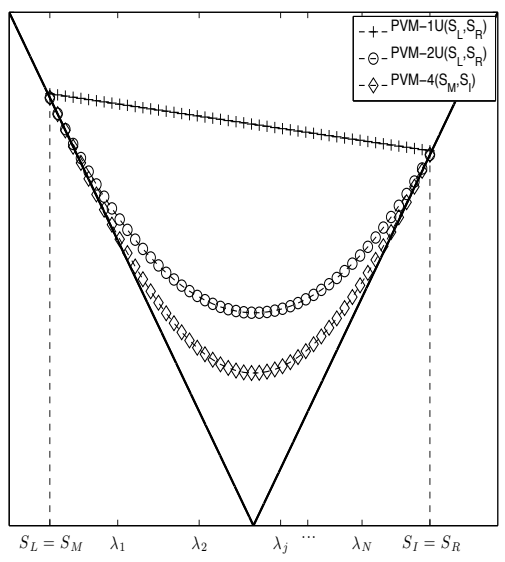

(b) two-waves PVM polynomials

FIG. 3.1. Graphics of the PVM polynomials.

where

$\mathcal{F}_{i+1 / 2}^{H L L}= \begin{cases}F\left(w_{i}\right) & \text { if } S_{L} \geq 0, \\ F^{H L L}=\frac{S_{R} F\left(w_{i}\right)-S_{L} F\left(w_{i+1}\right)+S_{R} S_{L}\left(w_{i+1}-w_{i}\right)}{S_{R}-S_{L}} & \text { if } S_{L} \leq 0 \leq S_{R}, \\ F\left(w_{i+1}\right) & \text { if } 0 \geq S_{R},\end{cases}$

where $S_{L}$ (respectively $S_{R}$ ) is an approximation of the minimum (respectively maximum) wave speed. One possible choice is to set $S_{L}=\lambda_{1, i+1 / 2}$ and $S_{R}=\lambda_{N, i+1 / 2}$, although some other different possibilities have been proposed in the bibliography, as, for example (see Davis [11])

$$
S_{L}=\min \left(\lambda_{1, i+1 / 2}, \lambda_{1, i}\right), \quad S_{R}=\max \left(\lambda_{N, i+1 / 2}, \lambda_{N, i+1}\right),
$$

where $\lambda_{i, 1}<\cdots<\lambda_{i, N}$ are the eigenvalues of matrix $A_{\Phi}\left(W_{i}, W_{i}\right)$.

HLL flux can be rewritten as a PVM- $1 \mathrm{U}\left(S_{L}, S_{R}\right)$ method by considering the polynomial

$$
P_{1}(x)=\alpha_{0}+\alpha_{1} x \quad \text { such as } P_{1}\left(S_{L}\right)=\left|S_{L}\right|, \text { and } P_{1}\left(S_{R}\right)=\left|S_{R}\right| .
$$

That is, $y=P_{1}(x)$ defines the straight line through points $\left(S_{L},\left|S_{L}\right|\right)$ and $\left(S_{R},\left|S_{R}\right|\right)$ (see Figure 3.1).

Some straightforward computations give

$$
\alpha_{0}=\frac{S_{R}\left|S_{L}\right|-S_{L}\left|S_{R}\right|}{S_{R}-S_{L}}, \quad \alpha_{1}=\frac{\left|S_{R}\right|-\left|S_{L}\right|}{S_{R}-S_{L}} .
$$

Let us suppose that the system (1.1) is conservative ( $B=0$ and $G=0)$, and let us define the conservative flux $\mathcal{F}_{i+1 / 2}=D_{i+1 / 2}^{-}+F\left(w_{i}\right)$, where $D_{i+1 / 2}^{-}$is given by (3.3), where $Q_{i+1 / 2}=P_{1}\left(\mathcal{L}\left(w_{i}, w_{i+1}\right)\right)$. Taking into account the expression of $\alpha_{0}$ and $\alpha_{1}$ in 
(3.3) gives

$$
\begin{aligned}
\mathcal{F}_{i+1 / 2}= & \left(F\left(w_{i}\right)\left(S_{R}+\left|S_{R}\right|-S_{L}-\left|S_{L}\right|\right)+F\left(w_{i+1}\right)\left(S_{R}-\left|S_{R}\right|-S_{L}+\left|S_{L}\right|\right)\right. \\
& \left.-\left(S_{R}\left|S_{L}\right|-S_{L}\left|S_{R}\right|\right)\left(w_{i+1}-w_{i}\right)\right) /\left(2 S_{R}-2 S_{L}\right),
\end{aligned}
$$

which is a compact definition of the numerical HLL flux $\mathcal{F}_{i+1 / 2}^{H L L}$ given in (3.12). Notice that PVM- $1 \mathrm{U}\left(S_{L}, S_{R}\right)$ is an upwind scheme, in the sense of Definition 3.1. Finally, observe that if $\lambda_{1, i+1 / 2}=-\lambda_{N, i+1 / 2}$, then PVM- $1 \mathrm{U}\left(S_{L}, S_{R}\right)$ coincides with PVM$0\left(S_{\text {Rus }}\right)$.

3.4. PVM-2 $\left(S_{0}\right)$ methods or FORCE type methods. In this subsection we study PVM methods corresponding to a second order polynomial of the form (see Figure 3.1)

$$
P_{2}(x)=\alpha_{0}+\alpha_{2} x^{2}, \text { such as } P_{2}\left(S_{0}\right)=S_{0}, P_{2}^{\prime}\left(S_{0}\right)=1,
$$

where $S_{0}$ is given by $(3.10)$.

Observe that, as $\alpha_{1}=0$, PVM-2 $\left(S_{0}\right)$ is not upwind in the sense of Definition 3.1. Some straightforward computations give

$$
\alpha_{0}=\frac{S_{0}}{2}, \quad \alpha_{2}=\frac{1}{2 S_{0}}
$$

Notice that if $S_{0}=S_{L F}$, then PVM-2 $\left(S_{L F}\right)$ coincides with FORCE scheme (see [30], [8]), while the other two can be seen as FORCE types schemes.

REMARK 3. GFORCE scheme can be obtained by imposing $P_{2}\left(S_{L F}^{m o d}\right)=S_{L F}^{m o d}$, $P_{2}^{\prime}\left(S_{L F}^{m o d}\right)=\frac{2 \gamma}{1+\gamma}$, which gives $\alpha_{0}=\frac{S_{L F}^{m o d}}{1+\gamma}$, and $\alpha_{2}=\frac{1}{S_{L F}^{m o d}} \frac{\gamma}{1+\gamma}$. It can be easily proved that $P_{2}(x)=\alpha_{0}+\alpha_{2} x^{2}$ given by (3.17) verifies that $P_{2}(x) \geq|x|$, $\forall x \in\left[\lambda_{1, i+1 / 2}, \lambda_{N, i+1 / 2}\right] \subset\left[-S_{0}, S_{0}\right]$, and therefore, the resulting methods are linearly $\mathrm{L}^{\infty}$-stable with the usual CFL condition.

REMARK 4. Notice that if $S_{0}=S_{L F}$ or $S_{0}=S_{L F}^{\text {mod }}$, then the coefficients $\alpha_{0}$ and $\alpha_{2}$ given in (3.17) depend on $\frac{\Delta x}{\Delta t}$ and PVM-2(S $\left.S_{0}\right)$ can be interpreted as a combination of Lax-Friedrichs and Lax-Wendroff schemes. In fact, this is the form in which FORCE and GFORCE schemes were introduced for conservative (see [30]) and nonconservative (see [8]) systems.

3.5. PVM-2U $\left(S_{M}, S_{m}\right)$ method. In [12] Degond et al. proposed a numerical scheme for conservative systems, that can be easily extended to nonconservative systems by considering a PVM method associated to the polynomial (see Figure 3.1)

$P_{2}(x)=\alpha_{0}+\alpha_{1} x+\alpha_{2} x^{2}$, such as $P_{2}\left(S_{m}\right)=\left|S_{m}\right|, P_{2}\left(S_{M}\right)=\left|S_{M}\right|, P_{2}^{\prime}\left(S_{M}\right)=\operatorname{sgn}\left(S_{M}\right)$,

where

$$
S_{M}= \begin{cases}\lambda_{1, i+1 / 2} & \text { if }\left|\lambda_{1, i+1 / 2}\right| \geq\left|\lambda_{N, i+1 / 2}\right| \\ \lambda_{N, i+1 / 2} & \text { if }\left|\lambda_{1, i+1 / 2}\right|<\left|\lambda_{N, i+1 / 2}\right|\end{cases}
$$

and

$$
S_{m}= \begin{cases}\lambda_{N, i+1 / 2} & \text { if }\left|\lambda_{1, i+1 / 2}\right| \geq\left|\lambda_{N, i+1 / 2}\right| \\ \lambda_{1, i+1 / 2} & \text { if }\left|\lambda_{1, i+1 / 2}\right|<\left|\lambda_{N, i+1 / 2}\right|\end{cases}
$$


It is easy to check that the coefficients are given by:

$$
\begin{aligned}
\alpha_{0} & =\frac{\left(S_{M}\right)^{2} S_{m}\left(\operatorname{sgn}\left(S_{m}\right)-\operatorname{sgn}\left(S_{M}\right)\right)}{\left(S_{m}-S_{M}\right)^{2}}, \\
\alpha_{1} & =\frac{S_{M}\left(\left|S_{M}\right|-\left|S_{m}\right|\right)+S_{m}\left(\operatorname{sgn}\left(S_{M}\right) S_{m}-S_{M} \operatorname{sgn}\left(S_{m}\right)\right)}{\left(S_{m}-S_{M}\right)^{2}}, \\
\alpha_{2} & =\frac{S_{m}\left(\operatorname{sgn}\left(S_{m}\right)-\operatorname{sgn}\left(S_{M}\right)\right)}{\left(S_{m}-S_{M}\right)^{2}} .
\end{aligned}
$$

It is easy to prove that $P_{2}\left(\lambda_{j, i+1 / 2}\right) \geq\left|\lambda_{j, i+1 / 2}\right| \forall j=1, \ldots, N$, so $\mathrm{PVM}-2 \mathrm{U}\left(S_{M}, S_{m}\right)$ is linearly $\mathrm{L}^{\infty}$-stable. Moreover, $P_{2}(x)$ verifies (3.7), thus PVM-2U $\left(S_{M}, S_{m}\right)$ is upwind. Finally, observe that if $\lambda_{1, i+1 / 2}=-\lambda_{N, i+1 / 2}$, then PVM-2U $\left(S_{M}, S_{m}\right)$ coincides with PVM-2( $\left.S_{\text {Rus }}\right)$.

3.6. PVM-4 $\left(S_{M}, S_{I}\right)$ and PVM-4 $\left(S_{0}\right)$ methods. In this section we introduce PVM methods defined by $4^{\text {th }}$ order polynomials given by (see Figure 3.1 )

$$
P_{4}(x)=\alpha_{0}+\alpha_{2} x^{2}+\alpha_{4} x^{4}, \text { such as } P_{4}\left(S_{M}\right)=\left|S_{M}\right|, \quad P_{4}\left(S_{I}\right)=S_{I}, \quad P_{4}^{\prime}\left(S_{I}\right)=1,
$$

where $S_{M}$ is defined by (3.19) and

$$
S_{I}=\left\{\begin{array}{cl}
\max _{2 \leq j \leq N}\left(\left|\lambda_{j, i+1 / 2}\right|\right) & \text { if }\left|\lambda_{1, i+1 / 2}\right| \geq\left|\lambda_{N, i+1 / 2}\right|, \\
\max _{1 \leq j \leq(N-1)}\left(\left|\lambda_{j, i+1 / 2}\right|\right) & \text { if }\left|\lambda_{1, i+1 / 2}\right|<\left|\lambda_{N, i+1 / 2}\right| .
\end{array}\right.
$$

It is easy to check that

$\alpha_{0}=\frac{\left|S_{M}\right|\left|S_{I}\right|\left(\left|S_{I}\right|+2\left|S_{M}\right|\right)}{2\left(\left|S_{I}\right|+\left|S_{M}\right|\right)^{2}}, \alpha_{2}=\frac{1}{2\left|S_{M}\right|}+\frac{\left|S_{M}\right|}{\left(\left|S_{I}\right|+\left|S_{M}\right|\right)^{2}}, \alpha_{4}=\frac{-1}{2\left|S_{M}\right|\left(\left|S_{I}\right|+\left|S_{M}\right|\right)^{2}}$.

Observe that $\alpha_{0}, \alpha_{2}$ and $\alpha_{4}$ are also well defined if $S_{M}=S_{I}=S_{0}$. In that case, the coefficients reduce to

$$
\alpha_{0}=\frac{3 S_{0}}{8}, \quad \alpha_{2}=\frac{3}{4 S_{0}}, \quad \alpha_{4}=\frac{-1}{8 S_{0}^{3}},
$$

and the method will be denoted as PVM-4 $\left(S_{0}\right)$, where $S_{0}$ is given by (3.10).

Concerning the stability of PVM- $4\left(S_{M}, S_{I}\right)$ method (PVM- $4\left(S_{0}\right)$ is a particular case) the following result can be proved:

Theorem 3.2. The numerical scheme PVM-4 $\left(S_{M}, S_{I}\right)$ is linearly $L^{\infty}$ stable under the usual CFL condition.

\section{Proof}

As $P_{4}(x)$ given by $(3.22)$ is symmetric with respect to 0 and $P_{4}\left(S_{M}\right)=\left|S_{M}\right|$, by the definition of $S_{I}$ it is enough to prove that $P_{4}(x) \geq x, \forall x \in\left[0, S_{I}\right]$.

Notice that, the first derivative of $P_{4}(x)$ is zero at $x=0$ and $x_{a}^{ \pm}= \pm \sqrt{\frac{-\alpha_{2}}{2 \alpha_{4}}}$, and the second derivative of $P_{4}(x)$ is zero at $x_{b}^{ \pm}= \pm \sqrt{\frac{-\alpha_{2}}{6 \alpha_{4}}}$.

Taking into account that $\left|S_{M}\right| \geq\left|S_{I}\right|$, then $\frac{-\alpha_{2}}{6 \alpha_{4}}=\frac{S_{I}^{2}+3 S_{M}^{2}+2\left|S_{M}\right|\left|S_{I}\right|}{6} \geq S_{I}^{2}$. Therefore $S_{I} \leq x_{b}^{+} \leq x_{a}^{+}$. We conclude that $P_{4}^{\prime}(x)$ is increasing in $\left[0, S_{I}\right]$. Moreover, 
as $P_{4}^{\prime}(0)=0, P_{4}(0)>0, P_{4}^{\prime}\left(S_{I}\right)=1$, and $P_{4}\left(S_{I}\right)=S_{I}$ then $P_{4}(x) \geq x$ for $x \in\left[0, S_{I}\right]$, what concludes the proof.

REMARK 5. The extension to high order of PVM schemes can be performed following the procedure described in [3]. The extension to $2 D$ systems is also straightforward following [4].

3.7. Numerical diffusion. In this section, a simple analysis of the numerical diffusion of the PVM schemes is performed. Let us consider the linear advection equation

$$
u_{t}+\lambda u_{x}=0, \quad \lambda>0 .
$$

It is easy to check that the numerical viscosity of methods PVM-l $\left(S_{0}\right), l=0,2,4$, when they are applied to equation (3.26) is given by

$$
\nu_{\mathcal{N}}=\frac{\Delta x}{2}\left(P_{l}(\lambda)-\gamma \lambda\right),
$$

where $\frac{\Delta t}{\Delta x} \lambda=\gamma$, and $P_{l}(x)$ are the polynomials associated to PVM-l $\left(S_{0}\right)$ methods, $l=0,2,4$ given by (3.9), (3.16), and (3.22) respectively. It is clear from (3.27) that $\nu_{\mathcal{N}}$ will be smaller if $P_{l}(\lambda), l=0,2,4$ is as small as possible. Notice that $P_{l}\left(S_{R u s}\right)=\lambda$ and $P_{l}\left(S_{L F}^{m o d}\right)=\lambda, l=0,2,4$ and $\mathrm{PVM}-\mathrm{l}\left(S_{R u s}\right)\left(\mathrm{PVM}-\mathrm{l}\left(S_{L F}^{\text {mod }}\right)\right.$ respectively $), l=0,2,4$ coincide with the CIR scheme (see [9]) for system (3.26). If $S_{0}=S_{L F}$, then

$$
P_{0}(\lambda)=\frac{\lambda}{\gamma}, P_{2}(\lambda)=\lambda \frac{1+\gamma^{2}}{2 \gamma}, P_{4}(\lambda)=\lambda \frac{3+6 \gamma^{2}-\gamma^{4}}{8 \gamma} .
$$

Therefore, $\nu_{\mathcal{N}}$ is minimum when $S_{0}=S_{R u s}$ or $S_{0}=S_{L F}^{m o d}$. If $S_{L F}$ is used, then PMV$4\left(S_{L F}\right)$ is the one with smallest numerical viscosity among the PVM-l $\left(S_{L F}\right)$ schemes, $l=0,2,4$. It is also easy to check that $P_{0}(x) \geq P_{2}(x) \geq P_{4}(x), x \in\left[-S_{0}, S_{0}\right]$. Finally, it is also easy to prove that PVM-l $\left(S_{0}\right), l=0,2,4$ with $S_{0}$ given by (3.10) are monotone schemes when they are applied to equation (3.26).

Concerning PVM-1U $\left(S_{L}, S_{R}\right)$ and PVM- $2 \mathrm{U}\left(S_{M}, S_{m}\right)$, is also easy to check that $P_{1}(x) \geq P_{2}(x), \forall x \in\left[\lambda_{1, i+1 / 2}, \lambda_{N, i+1 / 2}\right]$, where $P_{1}(x)$ and $P_{2}(x)$ are defined by (3.14) and (3.18), respectively. Therefore, the numerical viscosity of PVM- $2 \mathrm{U}\left(S_{M}, S_{m}\right)$ is smaller than PVM- $1 \mathrm{U}\left(S_{L}, S_{R}\right)$.

4. Numerical tests. Let us consider the two-fluid flow model of Pitman and Le [28] under the formulation given by Pelanti et al. in [27], where the friction terms are neglected

$$
\left\{\begin{array}{l}
\frac{\partial h_{f}}{\partial t}+\frac{\partial q_{f}}{\partial x}=0 \\
\frac{\partial q_{f}}{\partial t}+\frac{\partial}{\partial x}\left(\frac{q_{f}^{2}}{h_{f}}+\frac{g}{2} h_{f}^{2}\right)+g h_{f} \frac{\partial h_{s}}{\partial x}=-g h_{f} \frac{d b}{d x} \\
\frac{\partial h_{s}}{\partial t}+\frac{\partial q_{s}}{\partial x}=0 \\
\frac{\partial q_{s}}{\partial t}+\frac{\partial}{\partial x}\left(\frac{q_{s}^{2}}{h_{s}}+\frac{g}{2} h_{s}^{2}+g \frac{1-\rho}{2} h_{s} h_{f}\right)+\rho g h_{s} \frac{\partial h_{f}}{\partial x}=-g h_{s} \frac{d b}{d x}
\end{array}\right.
$$


In these equations, index $s$ ( $f$ respectively) makes reference to the solid (fluid respectively) phase. The granular fluid is assumed to occupy a straight channel with constant rectangular cross-section and constant width. The coordinate $x$ refers to the axis of the channel, $t$ is time, and $g$ is the acceleration due to gravity. $b(x)$ represents the fixed bottom topography and $\rho$ is the ratio of densities between the solid and fluid phase. The unknowns $h_{s}$ and $h_{f}$ are related to the total height of the granular fluid $h$ and the solid fraction $\psi$ by $h_{s}=\psi h$ and $h_{f}=(1-\psi) h$. The unknowns $q_{s}$ and $q_{f}$ represent the mass-flow of each phase and they are related with the mean velocity of each layer by $q_{k}=u_{k} h_{k}, k=s, f$.

System (4.1) can be written under the form of (1.1) by setting

$$
\begin{gathered}
w(x, t)=\left[\begin{array}{c}
h_{f}(x, t) \\
q_{f}(x, t) \\
h_{s}(x, t) \\
q_{s}(x, t)
\end{array}\right], \quad F(w)=\left[\begin{array}{c}
q_{f}^{2} \\
\frac{q_{f}}{h_{f}}+\frac{g}{2} h_{f}^{2} \\
q_{s} \\
\frac{q_{s}^{2}}{h_{s}}+\frac{g}{2} h_{s}^{2}+g \frac{1-r}{2} h_{f} h_{s}
\end{array}\right], \quad G(w)=\left[\begin{array}{c}
0 \\
-g h_{f} \\
0 \\
-g h_{s}
\end{array}\right], \\
B(w)=\left[\begin{array}{cccc}
0 & 0 & 0 & 0 \\
0 & 0 & g h_{f} & 0 \\
0 & 0 & 0 & 0 \\
g r h_{s} & 0 & 0 & 0
\end{array}\right], \quad H(x)=b(x) .
\end{gathered}
$$

Here, we have considered the Roe linearization based of the family of segments: given two states $W_{L}=\left[\begin{array}{lllll}h_{f}^{L} & q_{f}^{L} & h_{s}^{L} & q_{s}^{L} & b_{L}\end{array}\right]^{T}$ and $W_{R}=\left[\begin{array}{lll}h_{f}^{R} & q_{f}^{R} h_{s}^{R} q_{s}^{R} b_{R}\end{array}\right]^{T}$ the matrices $\mathcal{L}\left(w_{L}, w_{R}\right), B_{\Phi}\left(W_{L}, W_{R}\right)$, and the vector $G_{\Phi}\left(W_{L}, W_{R}\right)$ are defined by:

$$
\begin{gathered}
\mathcal{L}\left(w_{L}, w_{R}\right)=\left[\begin{array}{cccc}
0 & 1 & 0 & 0 \\
-\left(\hat{u}_{f}\right)^{2}+\left(\hat{c}_{f}\right)^{2} & 2 \hat{u}_{f} & 0 & 0 \\
0 & 0 & 0 & 1 \\
\frac{1-r}{2}\left(\hat{c}_{s}\right)^{2} & 0 & -\left(\hat{u}_{s}\right)^{2}+\left(\hat{c}_{s}\right)^{2}+\frac{1-r}{2}\left(\hat{c}_{f}\right)^{2} & 2 \hat{u}_{s}
\end{array}\right], \\
B_{\Phi}\left(W_{L}, W_{R}\right)=\left[\begin{array}{cccc}
0 & 0 & 0 & 0 \\
0 & 0 & \left(\hat{c}_{f}\right)^{2} & 0 \\
0 & 0 & 0 & 0 \\
r\left(\hat{c}_{s}\right)^{2} & 0 & 0 & 0
\end{array}\right] G_{\Phi}\left(W_{L}, W_{R}\right)=\left(\begin{array}{c}
0 \\
-\left(\hat{c}_{f}\right)^{2} \\
0 \\
-\left(\hat{c}_{s}\right)^{2}
\end{array}\right)
\end{gathered}
$$

where

$$
\hat{u}_{k}=\frac{\sqrt{h_{k}^{L}} u_{k}^{L}+\sqrt{h_{k}^{R}} u_{k}^{R}}{\sqrt{h_{k}^{L}}+\sqrt{h_{k}^{R}}}, \quad \hat{c}_{k}=\sqrt{g \frac{h_{k}^{L}+h_{k}^{R}}{2}}, u_{k}^{L}=\frac{q_{k}^{L}}{h_{k}^{L}}, \quad u_{k}^{R}=\frac{q_{k}^{R}}{h_{k}^{R}} \quad k=f, s .
$$

As in the case of two-layer shallow-water system (see [7] for example), a simple expression of the eigenvalues of matrix $A_{\Phi}\left(W_{L}, W_{R}\right)$ cannot be obtained and the hyperbolicity of system (4.1) is not always ensured. In [27] some bounds of the eigenvalues of $A_{\Phi}\left(W_{L}, W_{R}\right)$ are obtained and conditions ensuring the hyperbolicity of system (4.1) are derived. Here, we use the bounds derived in [27] to estimate the wave speeds to define the PMV schemes. Finally, Roe scheme for system (4.1) is constructed following [26]. In all the test cases considered here, the CFL parameter is set to $\gamma=0.9, r=0.5$, and $g=9.8$. Concerning the well-balancing properties, all the numerical schemes considered in this paper (first or high order) are well-balanced for the water at rest solution. 
4.1. Test 2.1. A 1D Riemann problem. Let us consider a Riemann problem, for which the initial condition consists in two constant states separated by an interface located at $x=0$. This test has been taken from [27]. The initial condition is defined in terms of the flow height and the solid volume fraction given by

$$
h(x, 0)=\left\{\begin{array}{ll}
3 & \text { if } x<0 \\
2 & \text { if } x>0,
\end{array} \quad \psi(x, 0)= \begin{cases}0.7 & \text { if } x<0 \\
0.4 & \text { if } x>0\end{cases}\right.
$$

and $u_{s}=u_{f}=0$. The computation is performed over the interval $[-5,5]$. Free boundary conditions are set.

Figure 4.1 shows the results for the flow depth $h$, the solid fraction $\psi$ at time $t=0.5$, computed with PVM-l $\left(S_{L F}\right), l=0,2,4$ and Roe scheme using $\Delta x=0.05$. A reference solution computed with Roe scheme for $\Delta x=1 / 640$ is also obtained. We can observe that the Riemann solution consists of a 1-rarefaction, 2-shock, 3-rarefaction, and a 4 -shock. It can also be observed that $\psi$ only varies across the internal waves. Concerning the quality of the numerical results, all numerical schemes provide the expected solution, being Roe scheme the less diffusive and Lax-Friedrichs the most diffusive (see Figure 4.1). Notice that in this test PVM-4 $\left(S_{L F}\right)$ and PVM-2 $\left(S_{L F}\right)$ approximately provide the same results.

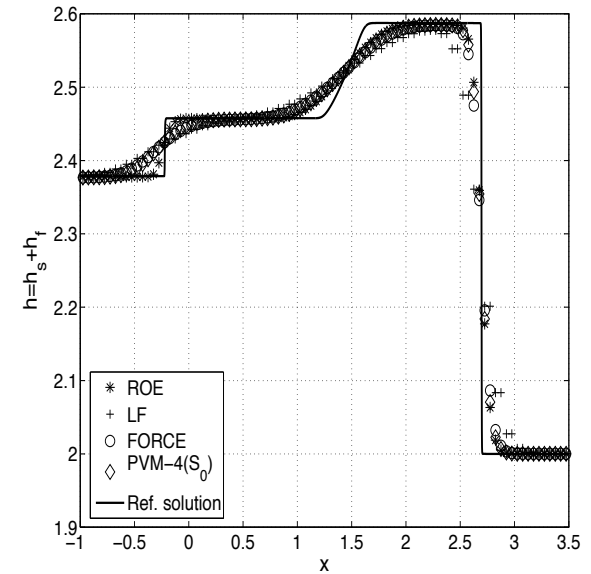

(a) Flow depth $h$ (zoom)

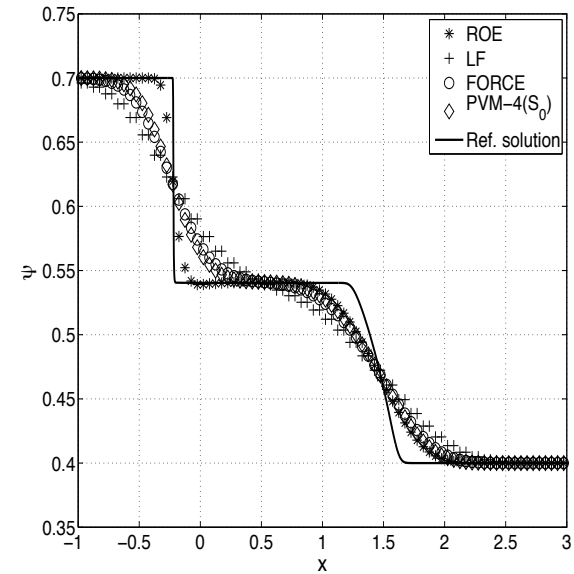

(b) Solid volume fraction $\psi$ (zoom)

Fig. 4.1. Test 2.1: Flow depth $h$ and solid volume fraction $\psi$ at time $t=0.5$. Comparison of Roe and PVM-l( $\left(S_{L F}\right), l=0,2,4$ schemes with the reference solution for $\Delta x=0.05$.

Figure 4.2 shows the results for flow depth $h$, the solid fraction $\psi$ at time $t=$ 0.5 , computed with PVM-1U $\left(S_{L}, S_{R}\right), \operatorname{PVM}-2 \mathrm{U}\left(S_{M}, S_{m}\right), \operatorname{PVM}-4\left(S_{M}, S_{I}\right)$, and Roe scheme using $\Delta x=0.05$. As expected, Roe scheme is the less diffusive and PVM$1 \mathrm{U}\left(S_{L}, S_{R}\right)$ the most diffusive. Notice that all schemes approximately coincide in the fastest waves while the major differences appear in the internal ones. Nevertheless, no significant differences can be observed between PVM-2U $\left(S_{M}, S_{m}\right)$ and $\operatorname{PVM}-4 \mathrm{U}\left(S_{M}, S_{I}\right)$.

Let us study in this test the influence in the numerical solutions of small changes in the family of paths. As in [6], we consider paths $\Phi_{h_{k}}^{\epsilon}\left(s ; W_{L}, W_{R}\right), k=f, s$, such as $h_{f}=\Phi_{h_{f}}^{\epsilon}\left(s ; W_{L}, W_{R}\right), h_{s}=\Phi_{h_{s}}^{\epsilon}\left(s ; W_{L}, W_{R}\right), s \in[0,1]$ is a parameterization of the 


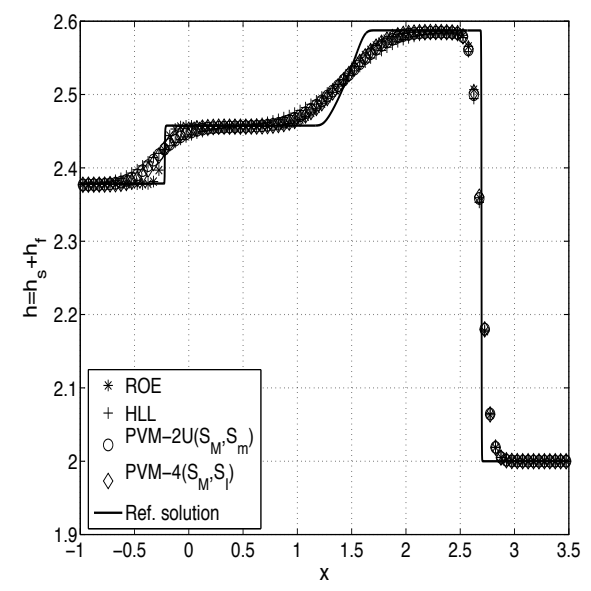

(a) Flow depth $h$

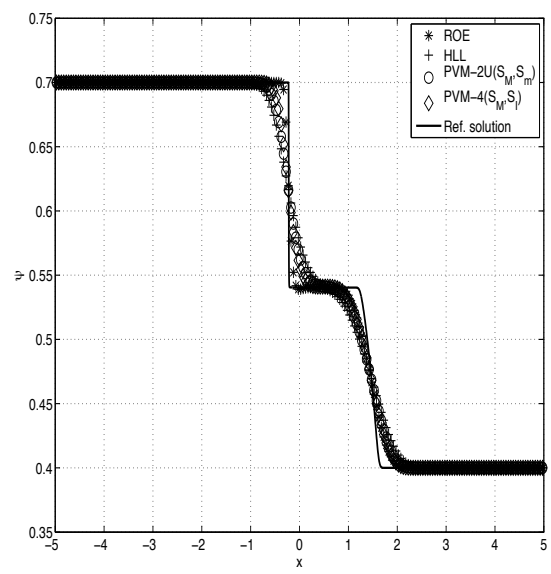

(b) Flow depth $h$ (zoom)

FIG. 4.2. Test 2.1: Flow depth $h$ and solid volume fraction $\psi$ at time $t=0.5$. Comparison of Roe, $P V M-1 U\left(S_{L}, S_{R}\right), P V M-2 U\left(S_{M}, S_{m}\right)$ and $P V M-4\left(S_{M}, S_{I}\right)$ schemes with the reference solution for $\Delta x=0.05$.

curve

$$
h_{s}=h_{s}^{L}+\left(\frac{h_{f}-h_{f}^{L}}{h_{f}^{R}-h_{f}^{L}}+\epsilon \frac{\left(h_{f}\right)^{2}-\left(h_{f}^{L}\right)^{2}}{\left(h_{f}^{R}\right)^{2}-\left(h_{f}^{L}\right)^{2}}\right) \frac{h_{s}^{R}-h_{s}^{L}}{1+\epsilon} .
$$

Observe that these paths can be seen as parabolic perturbations of the family of segments related to $h_{f}$ and $h_{s}$. Moreover, the family of segments are recovered for $\epsilon=0$.

The expresion for $B_{\Phi^{\epsilon}}$ can be computed easily and gives:

$$
B_{\Phi^{\epsilon}}\left(W_{L}, W_{R}\right)=\left[\begin{array}{cccc}
0 & 0 & 0 & 0 \\
0 & 0 & \left(\hat{c}_{f}^{\epsilon}\right)^{2} & 0 \\
0 & 0 & 0 & 0 \\
r\left(\hat{c}_{s}^{\epsilon}\right)^{2} & 0 & 0 & 0
\end{array}\right]
$$

where

$$
\begin{aligned}
\left(\hat{c}_{f}^{\epsilon}\right)^{2} & =\sqrt{g \frac{\left(h_{f}^{L}\right)^{2}(3+4 \epsilon)+2(3+2 \epsilon) h_{f}^{L} h_{f}^{R}+(3+4 \epsilon)\left(h_{f}^{R}\right)^{2}}{6(1+\epsilon)\left(h_{f}^{R}+h_{f}^{L}\right)}}, \\
\left(\hat{c}_{s}^{\epsilon}\right)^{2} & =\sqrt{g \frac{h_{f}^{R}\left((3+4 \epsilon) h_{s}^{L}+(3+2 \epsilon) h_{s}^{R}\right)+h_{f}^{L}\left((3+2 \epsilon) h_{s}^{L}+(3+4 \epsilon) h_{s}^{R}\right)}{6(1+\epsilon)\left(h_{f}^{R}+h_{f}^{L}\right)}} .
\end{aligned}
$$

Figure 4.3 shows the results for flow depth $h$ at time $t=0.5$, computed with $\operatorname{PVM}-2\left(S_{L F}\right)$ and PVM-2U $\left(S_{M}, S_{m}\right)$, for $\epsilon \in\{0,0.5,1.0\}$ using $\Delta x=0.001$. No significant differences on the position of the shocks can be observed for the different values of $\epsilon$ for the two PVM schemes. The same results can be observed for the solid fraction $\psi$ (see Figure 4.4), where the numerical solutions coincide up to the fourth decimal digit. So, at least, for this particular example, we can conclude that paths 
consisting on a parabolic perturbation of the family of segments give similar results, to those provided by the family of segments. Similar results are obtained in [6] for the two-layer shallow-water system.

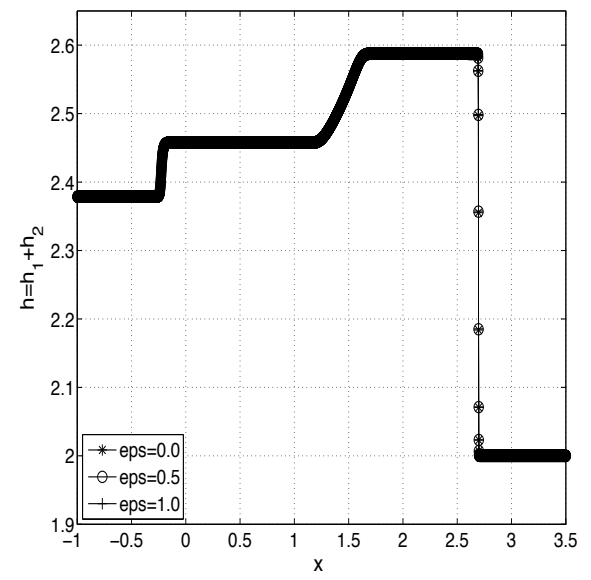

(a) PVM-2 $\left(S_{L F}\right)$ with $\epsilon \in\{0.0,0.5,1.0\}$

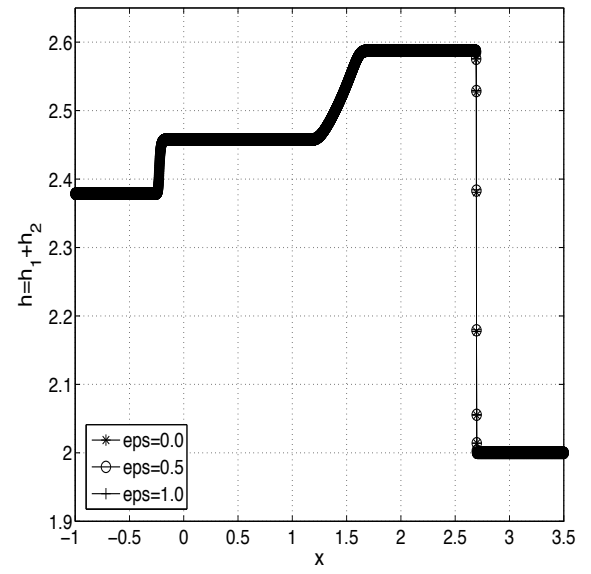

(b) PVM-2U $\left(S_{M}, S_{m}\right)$ with $\epsilon \in\{0.0,0.5,1.0\}$

FIG. 4.3. Test 2.1: Influence of the chosen path. $\epsilon \in\{0.0,0.5,1.0\}$. Flow depth $h$ at time $t=0.5$ computed with PVM-2(S $\left.S_{L F}\right)$ and PVM-2U $\left(S_{M}, S_{m}\right)$ for $\Delta x=0.001$.

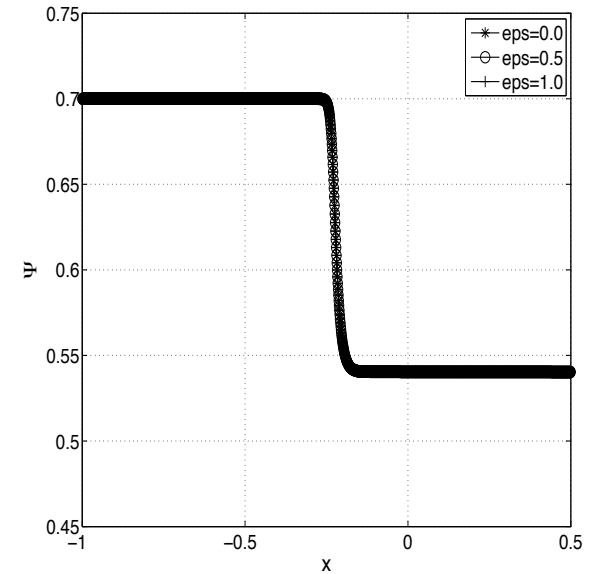

(a) PVM-2 $\left(S_{L F}\right)$ with $\epsilon \in\{0.0,0.5,1.0\}$

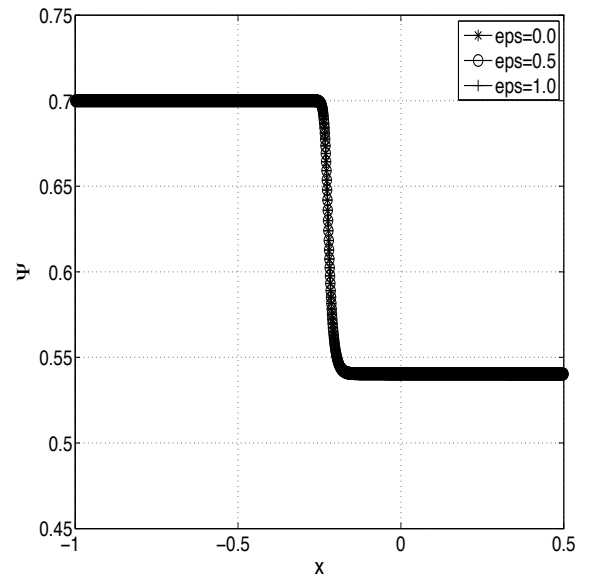

(b) PVM-2U $\left(S_{M}, S_{m}\right)$ with $\epsilon \in\{0.0,0.5,1.0\}$

FIG. 4.4. Test 2.1: Influence of the chosen path. $\epsilon \in\{0.0,0.5,1.0\}$. Solid fraction $\psi$ at time $t=0.5$ computed with PVM-2( $\left.S_{L F}\right)$ and $P V M-2 U\left(S_{M}, S_{m}\right)$ for $\Delta x=0.001$.

4.2. Test 2.2: Perturbation of a water at rest solution over a non flat bottom topography. Here, an experiment that is an extension of LeVeque's classical test [20] for one-layer shallow-water system with bottom topography is performed, that is, the behaviour of small perturbation of steady state solution corresponding 
to water at rest over a non flat bottom topography is studied. The computational domain is set to $[-0.9,1.0]$. Free flow boundary conditions are considered. The bottom topography is given by the function

$$
b(x)= \begin{cases}0.25(\cos (\pi(x-0.5) / 0.1)+1) & \text { if }|x-0.5|<0.1 \\ 0 & \text { otherwise }\end{cases}
$$

As initial condition we set $u_{s}(x, 0)=u_{f}(x, 0)=0$ and

$$
\begin{aligned}
& h(x, 0)= \begin{cases}1+10^{-3} & \text { if }-0.6<x<-0.5 \\
1-b(x) & \text { otherwise, }\end{cases} \\
& \psi(x, 0)= \begin{cases}0.6-10^{-3} & \text { if }-0.6<x<-0.5 \\
0.6 & \text { otherwise. }\end{cases}
\end{aligned}
$$

Figure 4.5 shows the results for the free surface $\eta=h+b$, the solid fraction $\psi$ at time $t=1.25$, computed with $\operatorname{PVM}-1\left(S_{L F}\right), l=0,2,4$ and Roe scheme using $\Delta x=0.01$. A reference solution computed with Roe scheme for $\Delta x=1 / 3200$ is also obtained. The initial perturbation is split into four waves, two going to the right and two to the left. The two waves going to the left have gone out and only the two going to the right are present in the domain. It can be seen that the right-going external wave has just passed over the obstacle at the bottom, and it has been partially reflected. It is well known that numerical schemes that are not well-balanced for the steady state at rest may produce in this kind of numerical test unphysical disturbances. All the numerical schemes considered here are well-balanced and they do not produce spurious oscillations. As in the previous numerical tests, all provide the expected solution, being Roe scheme the less diffusive and Lax-Friedrichs the most diffusive (see Figure 4.5), being PVM-4 $\left(S_{L F}\right)$ the less diffusive among the $\operatorname{PVM}-1\left(S_{L F}\right), l=0,2,4$ schemes.

Figure 4.6 shows the results for the free surface $\eta=h+b$, the solid fraction $\psi$ at time $t=1.25$, computed with PVM-1U $\left(S_{L}, S_{R}\right), \operatorname{PVM}-2 \mathrm{U}\left(S_{M}, S_{m}\right), \operatorname{PVM}-4\left(S_{M}, S_{I}\right)$, and Roe scheme using $\Delta x=0.01$. Again, Roe scheme is the less diffusive and PVM$1 \mathrm{U}\left(S_{L}, S_{R}\right)$ the most diffusive (see Figure 4.6. Notice that all schemes approximately coincide in the fastest waves, while major differences appear in the internal ones, as expected.

4.3. Test 2.3: $2 \mathrm{D}$ accuracy test. This test is inspired in the accuracy study presented in [14], where an unsteady two-dimensional analytical exact solution for the two-fluid flow model of Pitman and Le is obtained. The exact solution is computed as follows: first, one finds an exact stationary and rotationally symmetric solution of the governing PDE system supposing a constant bottom topography, that approaches a constant state as $r \rightarrow \infty$, in order to be compatible with periodic boundary conditions. Second, to make the test case unsteady, one uses the principle of Galilean invariance inherent in all governing equations based on Newtonian mechanics and superimposes a uniform velocity field to the solution found in step one.

Imposing rotational symmetry $\left(\frac{\partial}{\partial \phi}=0\right)$, the steady two-fluid Pitman and Le system in cylindrical coordinates $(r-\phi)$ reads as follows:

$$
\begin{aligned}
& g h_{s} \frac{\partial}{\partial r}\left(h_{s}+\frac{1+\rho}{2} h_{f}\right)+ \frac{1-\rho}{2} g h_{f} \frac{\partial}{\partial r} h_{s}=\frac{\left(u_{s}^{\phi}\right)^{2} h_{s}}{r}, \\
& g h_{f} \frac{\partial}{\partial r}\left(h_{s}+h_{f}\right)=\frac{\left(u_{f}^{\phi}\right)^{2} h_{f}}{r},
\end{aligned}
$$




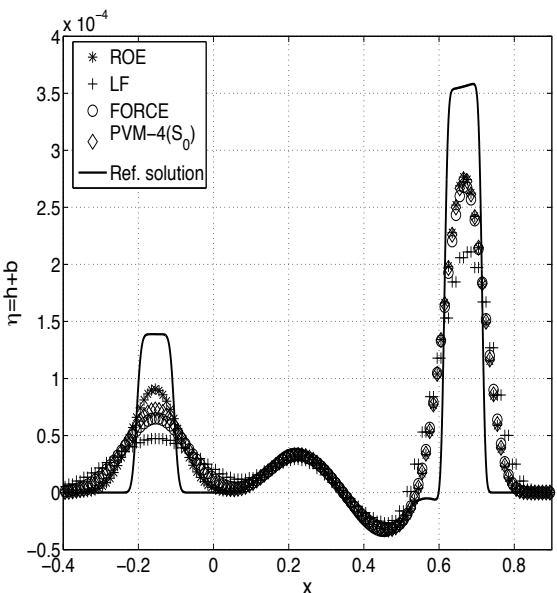

(a) Free surface $\eta=h+b$

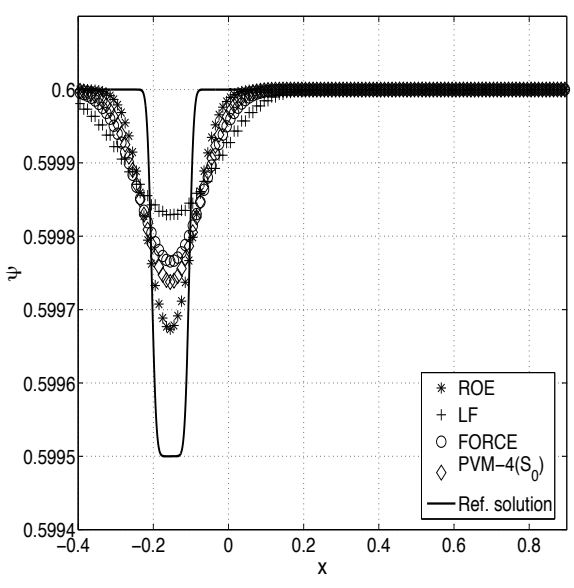

(b) Solid volume fraction $\psi$

FIG. 4.5. Test 2.2: Free surface $\eta=h+b$ and solid volume fraction $\psi$ at time $t=1.25$. Comparison of Roe and PVM-l( $\left.S_{L F}\right), l=0,2,4$ schemes with the reference solution for $\Delta x=0.01$.

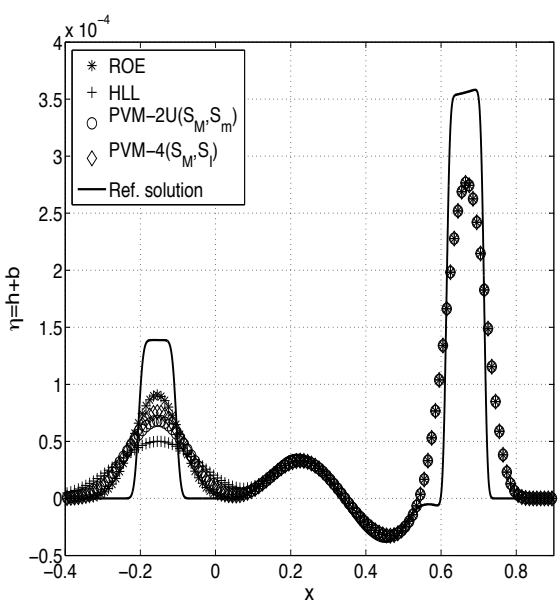

(a) Free surface $\eta=h+b$

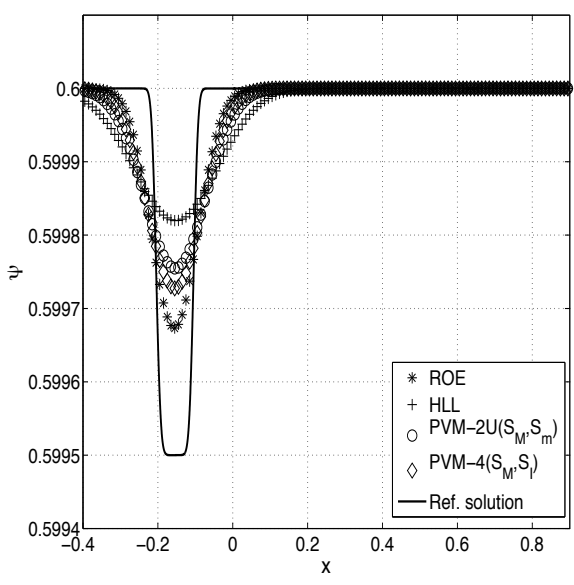

(b) Solid volume fraction $\psi$

FIG. 4.6. Test 2.2: Free surface $\eta=h+b$ and solid volume fraction $\psi$ at time $t=1.25$. Comparison of Roe, PVM-1U(S $\left.S_{L}, S_{R}\right), P V M-2 U\left(S_{M}, S_{m}\right)$ and PVM-4 $\left(S_{M}, S_{I}\right)$ schemes with the reference solution for $\Delta x=0.01$.

where $u_{k}^{\phi}(k=f, s)$ are the velocities in angular direction. Since we were not able to find a general solution of the nonlinear ODE system (4.6), we proceed in the following way: first, the velocity $u_{f}^{\phi}(r)$ is fixed and defined by $u_{f}^{\phi}=r u_{f, 0} e^{s_{f}\left(1-r^{2}\right)}$, where $u_{f, 0}$ and $s_{f}$ are two parameters to be defined. Note that $u_{f}^{\phi} \rightarrow 0$ as $r \rightarrow \infty$. Now, using the second equation of (4.6), the total high of the fluid $h(r)=h_{s}(r)+h_{f}(r)$ can be 
computed fixing the condition $h(0)=h_{0}$

$$
h(r)=\frac{4 g h_{0} s_{f}+u_{f, 0}^{2} e^{2 s_{f}}\left(1-e^{-2 s_{f} r^{2}}\right)}{4 g s_{f}}
$$

Next, $\psi(r)$ is prescribed, $\psi(r)=\psi_{0}+\psi_{1} e^{-2 s_{f} r^{2}}$ so that $h_{s}(r)$ and $h_{f}(r)$ can be computed from $h(r)$ and finally, $u_{s}^{\phi}$ is computed using the first equation of (4.6):

$u_{s}^{\phi}(r)=r \frac{\sqrt{2}}{2} \sqrt{\frac{u_{f, 0}^{2}\left(\left(\psi_{1}(\rho-1)+2 \psi_{0}\right) e^{2 s_{f}\left(1-r^{2}\right)}+\psi_{1}(3-\rho) e^{2 s_{f}\left(1-2 r^{2}\right)}\right)+\psi_{1} 4 g(\rho-1) h_{0} s_{f} e^{-2 s_{f} r^{2}}}{2+e^{-2 s_{f} r^{2}}}}$.

Note that $u_{s}^{\phi} \rightarrow 0$ as $r \rightarrow \infty$. In this test, $h_{0}=1, \rho=0.5, s_{f}=0.5, u_{f, 0}=0.5$, $\psi_{0}=0.5$ and $\psi_{1}=1 / 16$.

After having found the steady rotationally symmetric solution of the PDE, we can superimpose a uniform velocity field $\vec{u}=\left(\bar{u}_{x}, \bar{u}_{y}\right)^{T}=(5,5)^{T}$ to obtain the two dimensional velocity field

$$
u_{k, x}=\bar{u}_{x}-u_{k}^{\phi} \sin (\phi), u_{k, y}=\bar{u}_{y}+u_{k}^{\phi} \cos (\phi), k=f, s .
$$

We solve the 2D two-fluid Pitman and Le system with the initial solution computed here on the computational domain $[-5 ; 5] \times[-5 ; 5]$ with four periodic boundary conditions using a first and second order extension of PVM and Roe schemes to twodimensional domains, following the procedure described in [4]. Second order accuracy is achieved using a MUSCL type reconstruction and a second order Runge-Kutta TVD scheme (see [16] ). After one advection period $(t=2)$ the exact solution is given by the initial condition.

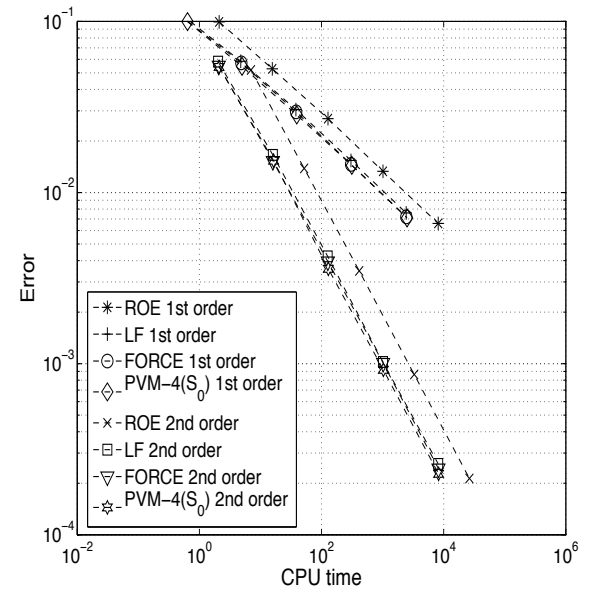

(a) CPU time versus errors: $1^{\text {st }}$ and $2^{\text {nd }}$ order one- (b) ratio of convergence: $1^{\text {st }}$ and $2^{\text {nd }}$ order onewave PVM and Roe schemes.

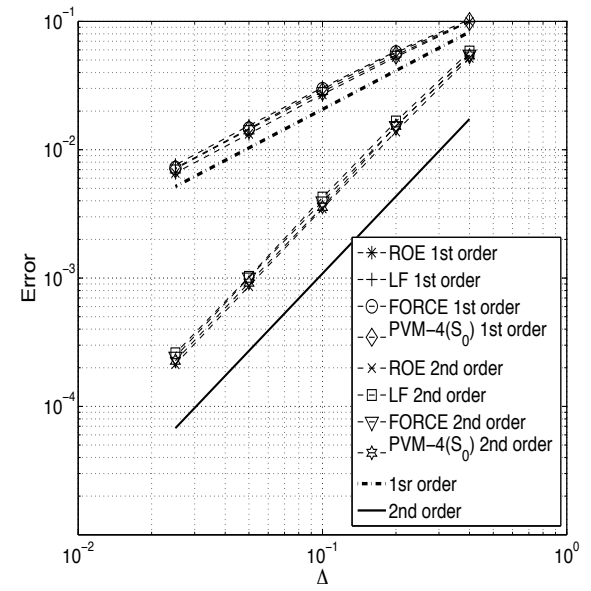

wave PVM and Roe schemes.

FIG. 4.7. Test 2.3: Efficiency and ratio of convergence of the one-wave PVM schemes: comparison with Roe scheme.

Figures 4.7(a) and 4.8(a) show the CPU time versus the error (computed with respect to the exact) for five meshes with increasing number of cells (from $\Delta x=$ 
$\Delta y=0.4$ to $\Delta x=\Delta y=0.025)$. Figure 4.7(a) shows the results for the PVM schemes defined with one wave (PVM-l $\left.\left(S_{L F}\right), l=0,2,4\right)$, and Figure 4.8(a) shows the results for the two-waves PVM schemes. Note that, for this example, first (respectively second order) PVM schemes are more efficient than first (respectively second order) Roe scheme. Concerning the ratio of convergence, second order schemes achieves the expected ratio of convergence (see Figures 4.7(b) and 4.8(b)). Second order PVM$4\left(S_{M}, S_{I}\right)$ scheme is the one that performs the best results among the PVM schemes concerning the efficiency, but no significant differences can be found with the others PVM schemes for this test.

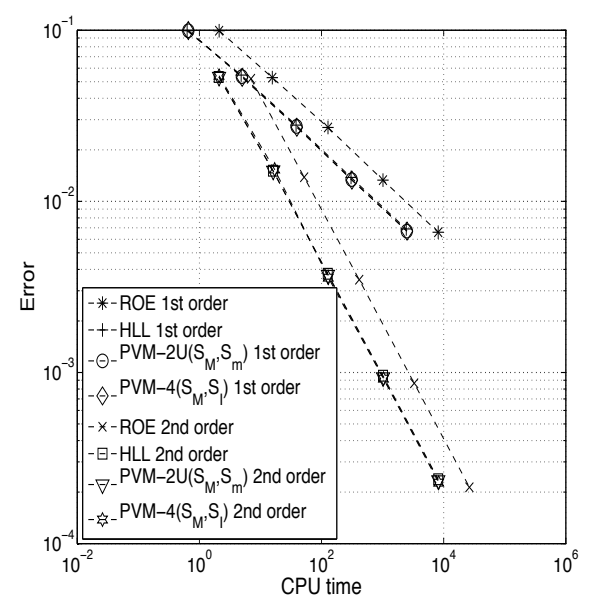

(a) CPU time versus errors: $1^{\text {st }}$ and $2^{\text {nd }}$ order one- (b) CPU time versus errors: $1^{\text {st }}$ and $2^{\text {nd }}$ order twowave PVM and Roe schemes.

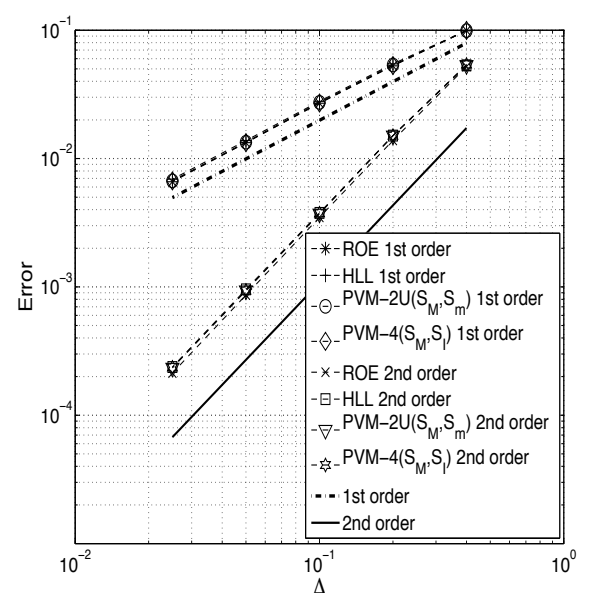

(b) CPU time versus errors: $1^{\text {st }}$
waves PVM and Roe schemes.

FiG. 4.8. Test 2.3: Efficiency and ratio of convergence of the two-waves PVM schemes: comparison with Roe scheme.

4.4. Test 2.4: a circular dam break problem. Finally, another $2 \mathrm{D}$ test corresponding to the Pitman-Le model has been performed using a first and second order extension of the PVM-2U $\left(S_{M}, S_{m}\right)$ scheme to two-dimensional domains, following the procedure described in [4].

Let us consider a $[-2,2] \times[-2,2]$ domain. The bottom function is given by $b(x, y)=0.5 e^{-0.5\left(x^{2}+y^{2}\right)}$. As initial condition we set $\vec{u}_{s}=\vec{u}_{f}=\overrightarrow{0}$ and

$$
\begin{gathered}
h(x, y, 0)= \begin{cases}1-b(x, y)+0.5 & \text { if } \sqrt{x^{2}+y^{2}} \leq 0.5, \\
1-b(x, y) & \text { otherwise }\end{cases} \\
\psi(x, y, 0)= \begin{cases}0.1 & \text { if } \sqrt{x^{2}+y^{2}} \leq 0.5, \\
0.9 & \text { otherwise. }\end{cases}
\end{gathered}
$$

Wall boundary conditions are set: $\vec{u}_{s} \cdot \vec{\eta}=\vec{u}_{f} \cdot \vec{\eta}=0$, where $\vec{\eta}$ is the unit normal vector to the boundaries. A mesh of $200 \times 200$ cells has been considered and a reference solution is computed using the first order Roe scheme with a mesh with $800 \times 800$ cells. Let us remark that the solution should maintain the radial symmetry of the initial condition until the waves interact with the boundaries. Figure 4.9 shows the evolution 
of the free surface $\eta=b+h$. Note that the first order scheme (left column) disturbs the radial symmetry of the solution, while the second order scheme produce a more accurate picture, as expected. Similar results can be observed in Figure 4.10, where the evolution of the solid volume fraction is shown.

Figure 4.11 shows the comparison of the free surface at two different 1D sections located at $x=0$ and $x=y$. Observe that no significant differences can be observed between the two sections when the second order scheme is used (right column), while some small differences can be found when using the first order scheme. Similar results are shown in Figure 4.12 for the solid volume fraction.

Concerning the efficiency, first and and second order PVM-2U $\left(S_{M}, S_{m}\right)$ schemes are faster than Roe. More precisely, a speed-up of 2.6 is obtained for the first order scheme and 4.7 for the second order scheme in comparison with Roe scheme. Figure 4.13 shows a comparison of the free surface computed with the first and second order Roe and PVM-2U $\left(S_{M}, S_{m}\right)$ schemes at the $1 \mathrm{D}$ section located at $x=0$. Note no significant differences can be found between the second order Roe and PVM-2U $\left(S_{M}, S_{m}\right)$ schemes. Similar results are obtained for the solid volume fraction.

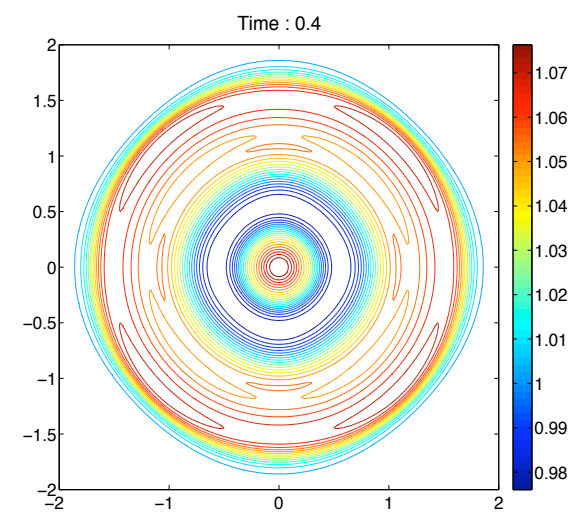

(a) $t=0.4$ s. First order PVM- $2 \mathrm{U}\left(S_{M}, S_{m}\right)$

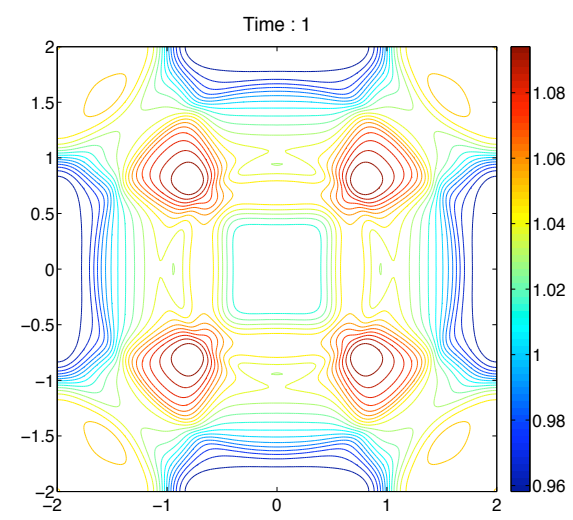

(c) $t=1 \mathrm{~s}$. First order PVM-2U $\left(S_{M}, S_{m}\right)$

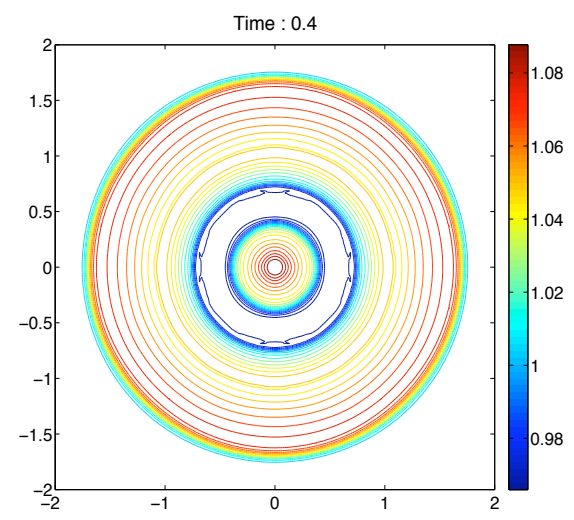

(b) $t=0.4 \mathrm{~s}$. Second order PVM-2U $\left(S_{M}, S_{m}\right)$

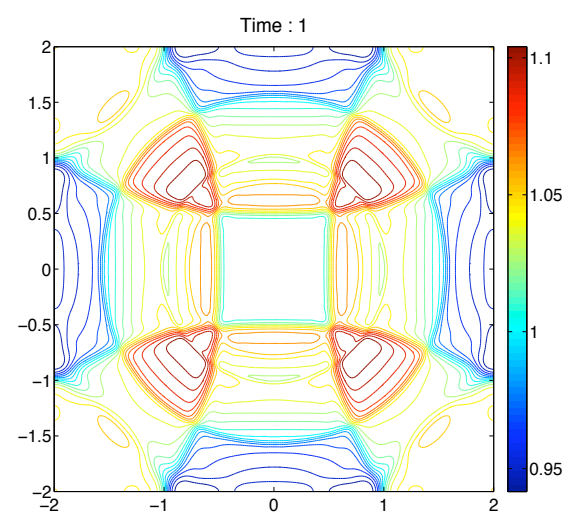

(d) $t=1 \mathrm{~s}$. Second order PVM-2U $\left(S_{M}, S_{m}\right)$

FIG. 4.9. Test 2.4: Evolution of the free surface $\eta=h+b$ : Comparison between first (left column) and second order (right column) PVM-2U( $\left.S_{M}, S_{m}\right)$ schemes 


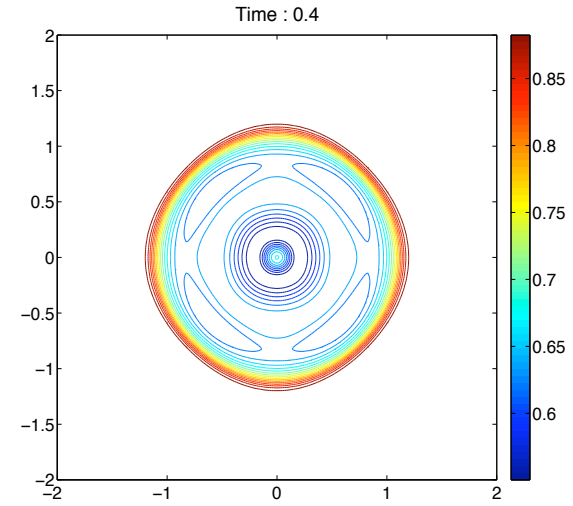

(a) $t=0.4$ s. First order PVM-2U $\left(S_{M}, S_{m}\right)$

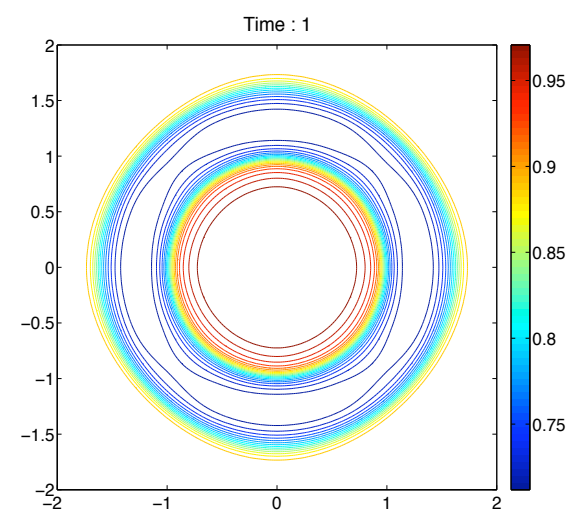

(c) $t=1 \mathrm{~s}$. First order PVM-2U $\left(S_{M}, S_{m}\right)$

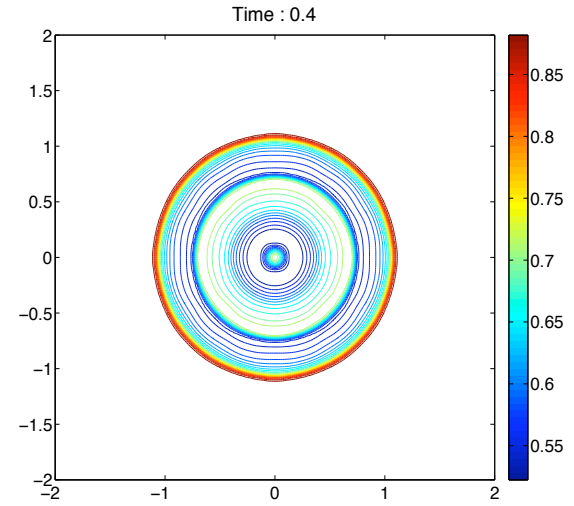

(b) $t=0.4 \mathrm{~s}$. Second order PVM-2U $\left(S_{M}, S_{m}\right)$

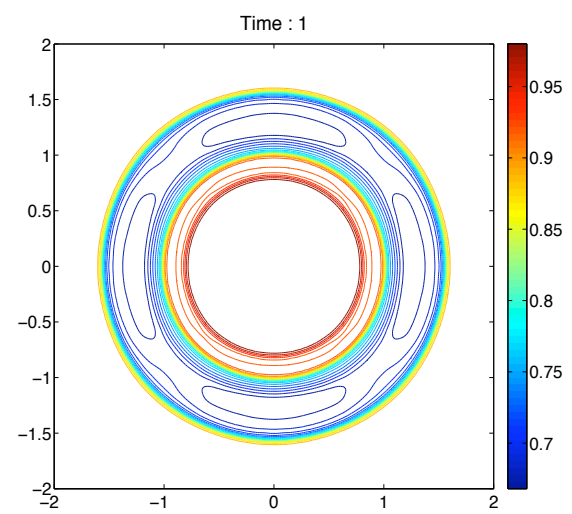

(d) $t=1 \mathrm{~s}$. Second order PVM-2U $\left(S_{M}, S_{m}\right)$

FIG. 4.10. Test 2.4: Evolution of the solid volume fraction $\psi$ : Comparison between first (left column) and second order (right column) PVM-2U( $\left.S_{M}, S_{m}\right)$ schemes

5. Conclusions. A family of first order path-conservative numerical schemes, named as PVM (Polynomial Viscosity Matrix), for balance laws or, more generally, for nonconservative hyperbolic systems has been introduced. They are defined in terms of viscosity matrices computed by a suitable polynomial evaluation of a Roe matrix. These methods have the advantage that they only need some information about the eigenvalues of the system to be defined, and no spectral decomposition of Roe Matrix is needed. As consequence, they are faster than Roe method. These methods can be seen as a generalization of the schemes introduced by Degond et al. in [12] for balance laws and nonconservative systems. Some well known solvers as Rusanov, Lax-Friedrichs, FORCE (see [30], [8]), GFORCE (see [31], [8]) or HLL (see [18]) are redefined under this form, and then some new solvers are also proposed. Well-balanced versions of these schemes for solving systems of conservation laws with source terms and/or nonconservative products can be easily obtained following the strategy described in [8]. A simple analysis of the numerical diffusion of PVM schemes are also performed. This analysis provides that PVM- $4\left(S_{0}\right)$ is the less diffusive among the PVM-l $\left(S_{0}\right), l=0,2,4$ and PVM- $4\left(S_{M}, S_{I}\right)$ is the less diffusive among those that 


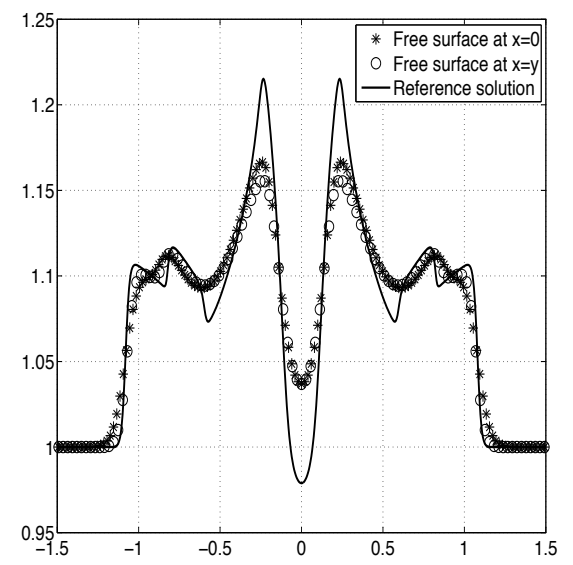

(a) $t=0.2$ s. First order PVM- $2 \mathrm{U}\left(S_{M}, S_{m}\right)$

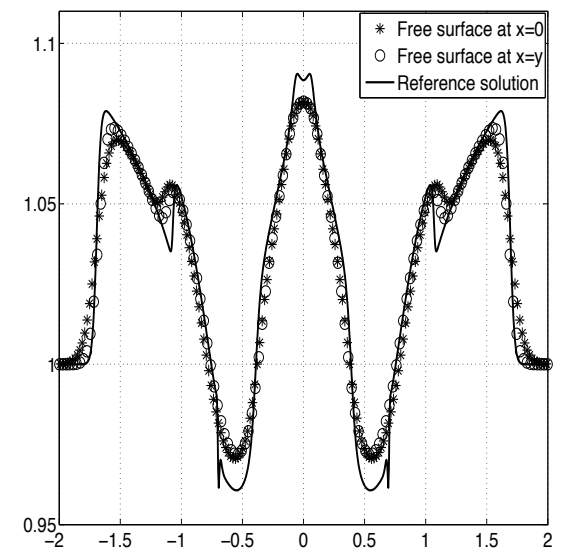

(c) $t=0.4$ s. First order PVM-2U $\left(S_{M}, S_{m}\right)$

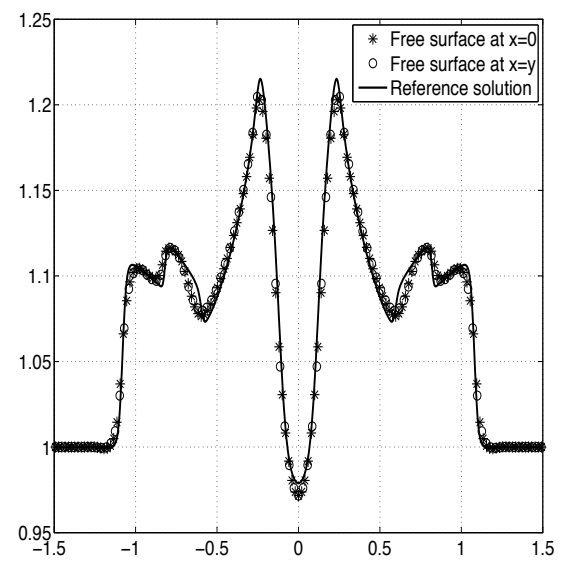

(b) $t=0.2 \mathrm{~s}$. Second order PVM-2U $\left(S_{M}, S_{m}\right)$

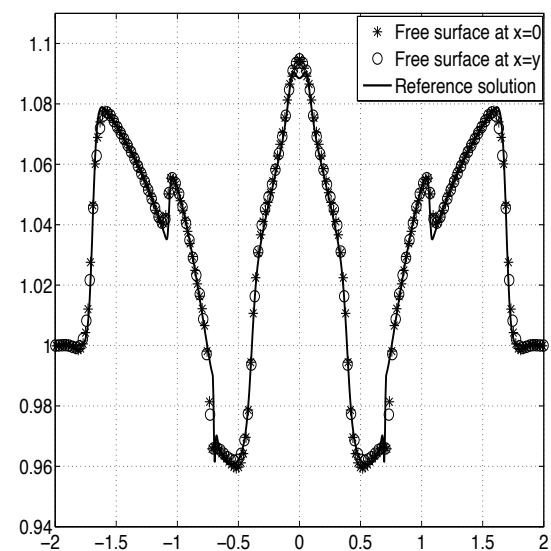

(d) $t=0.4 \mathrm{~s}$. Second order PVM-2U( $\left.S_{M}, S_{m}\right)$

FIG. 4.11. Test 2.4: Evolution of the free surface $\eta=h+b$ : Comparison between first (left column) and second order (right column) PVM-2U $\left(S_{M}, S_{m}\right)$ schemes at $1 D$ sections located at $x=0$ and $x=y$.

use information of two waves to be defined.

PVM schemes can been extended to high order by following the ideas developed in [3] and to two-dimensional systems following [4].

Concerning the quality of he numerical solutions, the best first order numerical scheme is, of course, Roe followed by PVM- $4\left(S_{M}, S_{I}\right)$. PVM- $2 \mathrm{U}\left(S_{M}, S_{m}\right)$ is an interesting choice as it is an upwind scheme and provides as good results as PVM$4\left(S_{M}, S_{I}\right)$. The high order extensions are similar regardless of the first order scheme considered. Therefore, PVM schemes are an excellent alternative to Roe methods to approximate time-dependent solutions when the calculation of the eigenvalues has to be numerically performed.

Finally, concerning the influence of the path on the numerical solutions, it seems 


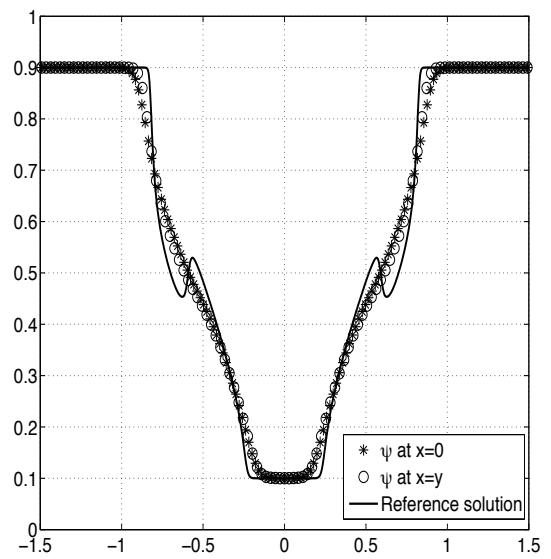

(a) $t=0.2$ s. First order PVM- $2 \mathrm{U}\left(S_{M}, S_{m}\right)$

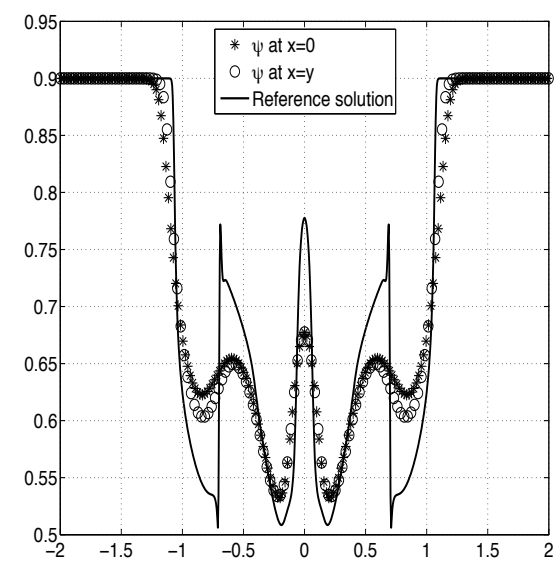

(c) $t=0.4$ s. First order PVM- $2 \mathrm{U}\left(S_{M}, S_{m}\right)$

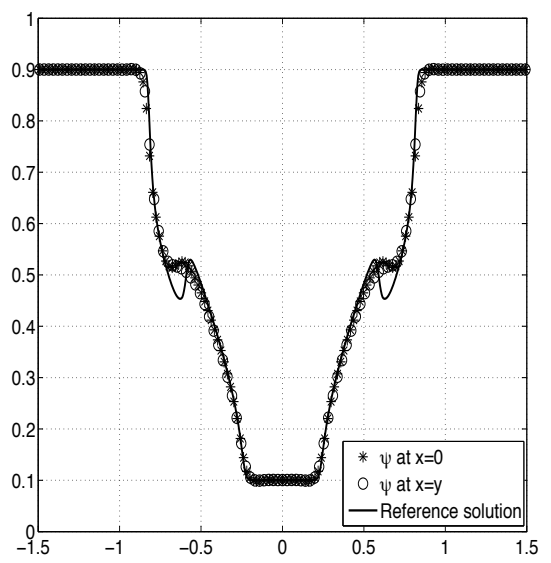

(b) $t=0.2 \mathrm{~s}$. Second order PVM-2U $\left(S_{M}, S_{m}\right)$

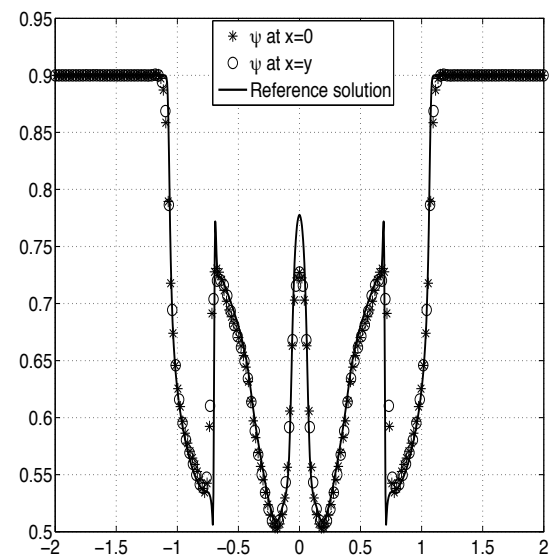

(d) $t=0.4 \mathrm{~s}$. Second order PVM-2U $\left(S_{M}, S_{m}\right)$

FIG. 4.12. Test 2.4: Evolution of the solid volume fraction $\psi$ : Comparison between first (left column) and second order (right column) PVM-2U(SM,$\left.S_{m}\right)$ schemes at $1 D$ sections located at $x=0$ and $x=y$.

that paths that are obtained by a parabolic perturbation of segments gives similar results to those provided by the family of segments for the Pitman-Le model.

\section{REFERENCES}

[1] Abgrall R., Karni S., A comment on the computation of non-conservative products. J. Comput. Phys., 45, 382-403, 2010.

[2] A. Bermúdez, M.E. Vázquez. Upwind methods for hyperbolic conservation laws with source terms. Computers and Fluids, 23(8): 1049-1071, 1994.

[3] M.J. Castro, J.J. Gallardo, and C. Parés. High order finite volume schemes based on reconstruction of states for solving hyperbolic systems with nonconservative products. Applications to shallow-water systems. Math. Comp. 75:1103-1134, 2006. 


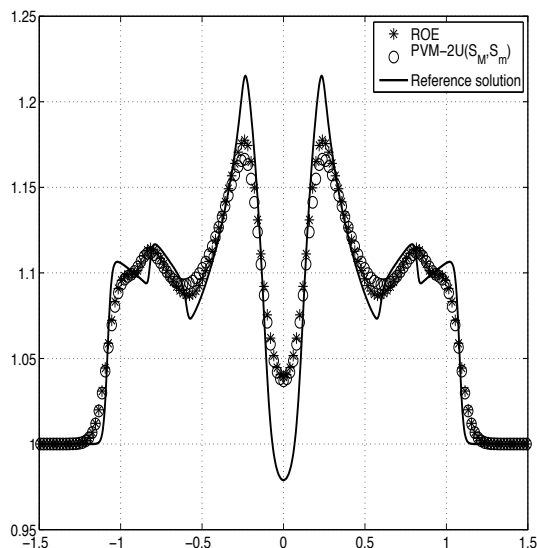

(a) $t=0.2 \mathrm{~s}$. First order schemes

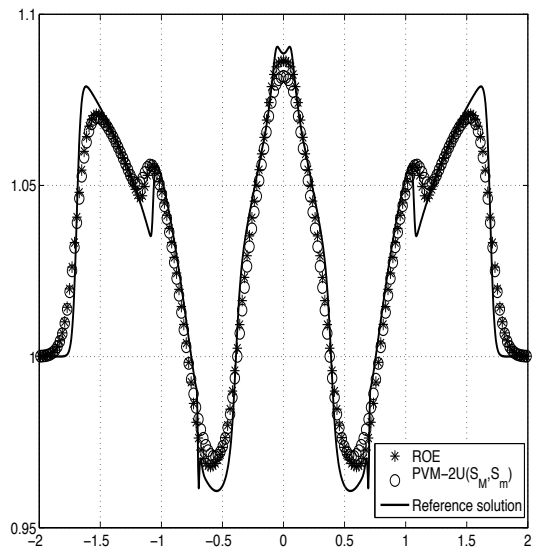

(c) $t=0.4 \mathrm{~s}$. First order schemes

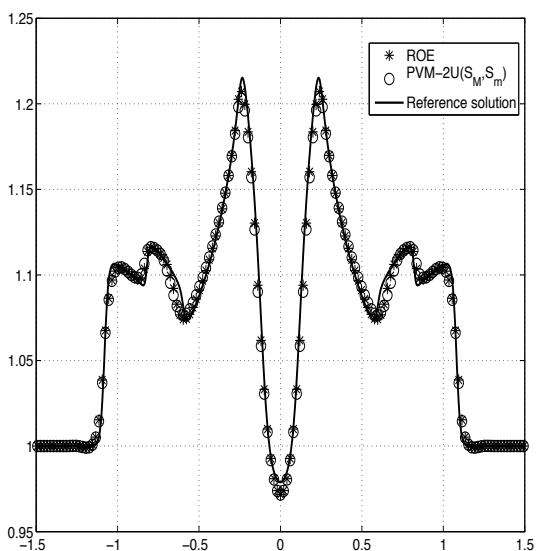

(b) $t=0.2 \mathrm{~s}$. Second order schemes

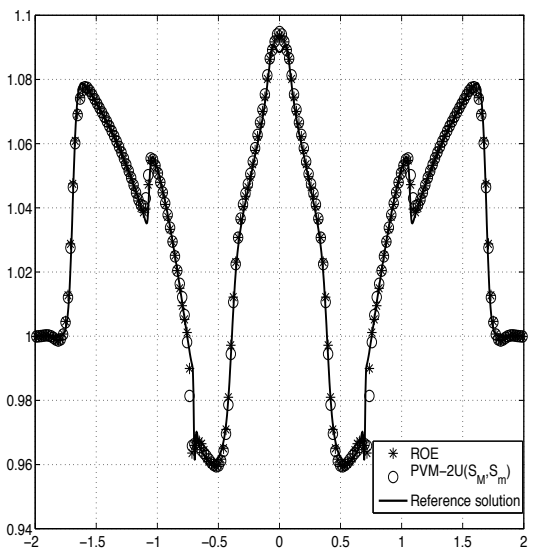

(d) $t=0.4 \mathrm{~s}$. Second order schemes

FIG. 4.13. Test 2.4: Evolution of the free surface $\eta=b+h$ : Comparison between first (left column) and second (right column) order Roe and PVM-2U $\left(S_{M}, S_{m}\right)$ schemes at $1 D$ section located at $x=0$.

[4] M.J. Castro, E.D. Fernández, A.M.Ferreiro, J.A. García, and C. Parés High order extensions of Roe schemes for two dimensional nonconservative hyperbolic systems. Jour. Sci. Comp. 39: 67-114, 2009.

[5] M.J. Castro, A.M.Ferreiro, J.A. García, J.M. González, J. Macías, C. Parés and M.E. Vázquez On the numerical treatment of wet/dry fronts in shallow flows: applications to one-layer and two-layer systems. Math. Comp. Model. 42 (3-4): 419-439, 2005.

[6] M.J. Castro, P.G. LeFloch, M.L. Muñoz, and C. Parés. Why many theories of shock waves are necessary: Convergence error in formally path-consistent schemes. Jour. Comp. Phys. 3227: 8107-8129, 2008.

[7] M.J. Castro, J. Macías, C. Parés. A Q-Scheme for a class of systems of coupled conservation laws with source term. Application to a two-layer 1-D shallow water system. Math. Mod. Num. Anal. 35(1): 107-127, 2001.

[8] M.J. Castro, A. Pardo, C. Parés, and E.F. Toro. On some fast well-balanced first order 
solvers for nonconservative systems. Math. Comp, 79 (271): 1427-1472, 2010.

[9] R. Courant, E. Isaacson and M. Rees On the solution of nonlinear hyperbolic differential equations by finite differences. Comm. Pure Appl. Math. 5, 243255, 1952.

[10] G. Dal Maso, P.G. LeFloch, F. Murat. Definition and weak stability of nonconservative products. J. Math. Pures Appl. 74: 483-548, 1995.

[11] S. F. Davis. Simplified Second-Order Godunov-Type Methods. SIAM J. Sci. Stat. Comput., 9:445473, 1988.

[12] P. Degond, P.F. Peyrard, G. Russo, Ph. Villedieu. Polynomial upwind schemes for hyperbolic systems. C. R. Acad. Sci. Paris 1 328, 479-483, 1999.

[13] M. Dumbser, A. Hidalgo, M.J. Castro, C. Parés, E.F. Toro, FORCE schemes on unstructured meshes II: Non-conservative hyperbolic systems. Comp. Meth. Appl. Mech. Eng. 199, 625-647, 2010.

[14] M. Dumbser, M.J. Castro, C. Parés, E. F. Toro. ADER schemes on unstructured meshes for nonconservative hyperbolic systems: Applications to geophysical flows. Computers \&; Fluids, 38(9): 1731-1748, 2009.

[15] B. Einfeldt. On Godunov-type methods for gas dynamics. SIAM J. Numer. Anal. 25: 294-318, 1988.

[16] S. Gottlieb And C.-W. Shu, Total variation diminishing Runge-Kutta schemes. Math. Comp. 67,73-85, 1998.

[17] A. Harten, J.M. Hyman. Self-adjusting grid methods for one-dimensional hyperbolic conservation laws. J. Comp. Phys. 50: 235-269, 1983.

[18] A. Harten, P.D. Lax, B. van Leer B. On Upstream Differencing and Godunov Type Schemes for Hyperbolic Conservation Laws. SIAM Review, 25(1):3561, 1983.

[19] T.Y. Hou, P.G. LeFloch. Why nonconservative schemes converge to wrong solutions: error analysis. Math. of Comput. 62: 497-530, 1994.

[20] R. LeVeque. Balancing source terms and flux gradients in high-resolution Godunov methods: the quasi-steady wave-propagation algorithm. J. Comp. Phys. 146: 346$365,1998$.

[21] T. Morales de Luna, M.J. Castro, C. Parés, E.D. Fernández-Nieto. On a shallow water model for the simulation of turbidity currents. Comm. Comp. Phys. 6, 848-882, 2009.

[22] M.L. Muñoz-Ruiz, C. Parés, Godunov method for nonconservative hyperbolic systems, Math. Model. Numer. Anal. 41, 169-185, 2007.

[23] M.L. Muñoz, C. Parés.On the convergence and well-balanced property of pathconservative numerical schemes for systems of balance laws. Submitted to Jour. Sci. Comp., 2010.

[24] C. Parés. Numerical methods for nonconservative hyperbolic systems: a theoretical framework. SIAM J. Num. Anal. 44(1): 300-321, 2006.

[25] C. Parés and M.L. Muñoz Ruíz. On some difficulties of the numerical approximation of nonconservative hyperbolic systems. Boletín SEMA, 47:23-52, 2009.

[26] C. Parés, M.J. Castro. On the well-balance property of Roe's method for nonconservative hyperbolic systems. Applications to Shallow-Water Systems. M2AN, Vol. 38(5): 821-852, 2004.

[27] M. Pelanti, F. Bouchut, and A. Mangeney. A Roe-type scheme for two-phase shallow granular flows over variable topography. M2AN, Vol. 42: 851-885, 2008.

[28] E.B.Pitman and L. Le.A two-fluid model for avalanche and debris flows. Phil. Trans. R. Soc. A, 363: 1573-1601, 2005.

[29] P.L. Roe. Approximate Riemann solvers, parameter vectors and difference schemes. J. Comp. Phys. 43: 357-371, 1981.

[30] E.F. Toro, S.J. Billett. Centred TVD schemes for hyperbolic conservation laws. IMA Journal of Numerical Analysis, Vol. 20, pp 47-79, 2000.

[31] E.F. Toro, V.A. Titarev. MUSTA fluxes for systems of conservation laws. J. Comput. Phys. 216(2): 403429, 2006.

[32] I. Toumi. A weak formulation of Roe approximate Riemann solver. J. Comp. Phys. 102(2): 360-373, 1992. 